\title{
Description of odd-mass nuclei within the interacting boson-fermion model based on the Gogny energy density functional
}

\author{
K. Nomura, ${ }^{1,2}$ R. Rodríguez-Guzmán, ${ }^{3}$ and L. M. Robledo ${ }^{4}$ \\ ${ }^{1}$ Physics Department, Faculty of Science, University of Zagreb, HR-10000 Zagreb, Croatia \\ ${ }^{2}$ Center for Computational Sciences, University of Tsukuba, Tsukuba 305-8577, Japan \\ ${ }^{3}$ Physics Department, Kuwait University, 13060 Kuwait, Kuwait \\ ${ }^{4}$ Departamento de Física Teórica, Universidad Autónoma de Madrid, E-28049 Madrid, Spain \\ (Received 16 May 2017; revised manuscript received 26 June 2017; published 21 July 2017)
}

\begin{abstract}
Spectroscopic properties of odd-mass nuclei are studied within the framework of the interacting bosonfermion model (IBFM) with parameters based on the Hartree-Fock-Bogoliubov (HFB) approximation. The parametrization D1M of the Gogny energy density functional (EDF) was used at the mean-field level to obtain the deformation energy surfaces for the considered nuclei in terms of the quadrupole deformations $(\beta, \gamma)$. In addition to the energy surfaces, both single-particle energies and occupation probabilities were used as a microscopic input for building the IBFM Hamiltonian. Only three strength parameters for the particle-boson-core coupling are fitted to experimental spectra. The IBFM Hamiltonian is then used to compute the energy spectra and electromagnetic transition rates for selected odd-mass Eu and Sm nuclei as well as for ${ }^{195} \mathrm{Pt}$ and ${ }^{195} \mathrm{Au}$. A reasonable agreement with the available experimental data is obtained for the considered odd-mass nuclei.
\end{abstract}

DOI: 10.1103/PhysRevC.96.014314

\section{INTRODUCTION}

The study of the nuclei with odd number of protons $Z$ and/or neutrons $N$ has been a recurring theme of great interest in low-energy nuclear physics. While a wealth of new spectroscopic data have been produced experimentally for odd nuclei, especially in medium- and heavy-mass regions, a microscopic description of odd nuclei represents one of the most challenging problems on the theoretical side. One of the reasons is the treatment of pairing: this type of correlation couples protons and neutrons in even-even nuclei to form Cooper pairs similar to the ones of the phenomenon of superfluidity in condensed matter physics. The presence of such Cooper pairs influences in an important way nuclear dynamics, determining basic parameters of vibrational and rotational spectra [1]. The treatment of pairing correlations in those cases relies on the Hartree-Fock-Bogoliubov (HFB) mean field theory characteristic of even-even nuclei. On the other hand, the description of odd- $Z$ and/or odd- $N$ nuclei requires one to describe at the same time the Cooper pairs and the unpaired nucleon, that is boson-like (Cooper pairs) and fermionic (unpaired nucleon) degrees of freedom. The treatment of both degrees of freedom requires extending the HFB theory to include blocking, with the subsequent complications arising by the explicit breaking of time reversal invariance [1,2]. Another unwanted consequence is the need to explicitly treat both the collective and single-particle degrees of freedom on the same footing.

The above difficulties are not a problem for the shell model (SM) [3], but the exceedingly large size of shell model configuration spaces in medium-mass and heavy nuclei and/or open shell nuclei makes it impractical for the purpose of describing odd nuclei in those cases.

On the other hand, the theoretical approaches based on the energy density functional (EDF) method [4] allow for a global description of many low energy properties of nuclei all over the nuclide chart, including medium-mass and heavy ones. Although not as common as in the even-even case, a number of calculations have been made within the EDF framework at the mean-field level for odd-mass systems (see [5-8] for some recent examples with the Gogny force). In the EDF framework, a proper description of excited states requires the inclusion of dynamical correlations associated with the restoration of broken symmetries and fluctuations via the symmetry-projected configuration mixing calculation within the generator coordinate method (GCM). In Ref. [9], the GCM framework was extended to the odd-mass systems by explicitly taking into account the breaking of time-reversal symmetry. Nevertheless, the practical applications of this approach to medium-heavy and heavy nuclei are computationally demanding, and so far calculations have been limited to very light-mass systems $[9,10]$.

Given the difficulties encountered with the two major theoretical approaches to nuclear structure mentioned earlier, i.e., SM and EDF-GCM frameworks, it is worth to consider alternative theoretical approaches to odd nuclei. Among them we can mention the various extensions of the particlevibration coupling scheme [1,11-15] and also algebraic based approaches [16-20], that provide a computationally more economic as well as straightforward description of the odd nuclei in all mass regions from light to heavy nuclei. Along this direction, one of the present authors has recently developed a method [21] to calculate the spectroscopic properties of odd-mass nuclei, which is based on the EDF framework combined with the particle-boson-core coupling scheme, i.e., the interacting boson-fermion model [17]. In this proposal [21], the energy surface of an even-even nucleus given as a function of the shape parameters describing quadrupole deformation $(\beta, \gamma)$, along with single-particle energies and occupation probabilities of the odd nucleons computed within the self-consistent mean-field method (based on the relativistic EDF with the parametrization DD-PC1 [22]), are used as a 
microscopic input to determine the parameters of an IBFM Hamiltonian. Only the strength parameters of the bosonfermion coupling term in the IBFM Hamiltonian have been determined so as to fit the selected experimental data. The validity of the method was confirmed in the axially deformed odd-mass $\mathrm{Eu}$ isotopes [21] and further applied to study shape phase transitions between nearly spherical and axially deformed shapes in the odd-mass $\mathrm{Eu}$ and Sm isotopes [23], and between nearly spherical and $\gamma$-soft shapes in the mass $A \approx 130$ region [24].

In this work, we apply the method of Ref. [21] to describe spectroscopic properties of selected odd-mass nuclei based on the Gogny EDF. Specifically, we consider the odd-mass isotopes ${ }^{149-155} \mathrm{Eu}$ and ${ }^{149-155} \mathrm{Sm}$ as well as the ${ }^{195} \mathrm{Pt}$ and the ${ }^{195} \mathrm{Au}$ nuclei. Their associated even-even core nuclei, ${ }^{148-154} \mathrm{Sm}$ and ${ }^{194} \mathrm{Pt}$, are good examples of axially deformed and $\gamma$-soft nuclei, respectively. By studying the same isotopes $\left({ }^{149-155} \mathrm{Eu}\right.$ and $\left.{ }^{149-155} \mathrm{Sm}\right)$ as in Refs. [21,23], we demonstrate that the IBFM Hamiltonian based on the Gogny EDF describes the low-lying states in odd-mass nuclei at the same level of accuracy as in the earlier studies of [21,23] based on the relativistic EDF. The Gogny EDF is a successful member of the class of nonrelativistic EDFs. It has been used in many nuclear structure and reaction theory calculations all over the nuclide chart with great success. Its accuracy in describing experimental data is at the level of the one obtained by performing modern relativistic EDF calculations. By comparing the results within the two major classes of EDF, i.e., nonrelativistic and relativistic EDFs, we demonstrate the validity of the mapping procedure of Ref. [21]. Furthermore, the addition of the $\gamma$-soft nuclei, ${ }^{195} \mathrm{Pt}$ and ${ }^{195} \mathrm{Au}$, which were not included in Refs. [21,23], further confirms the robustness of the procedure.

We employ the parametrization D1M [25] of the Gogny EDF. A number of previous studies have demonstrated that the D1M set, apart from being much more efficient in the description of binding energies than the more traditional and extensively tested D1S parametrization [26], keeps the same predictive power as D1S in the description of other nuclear properties such as excitation energies or transition strengths. Nevertheless, we have also carried out part of the calculations with D1S and confirmed the striking similarities between the two sets of results. As a consequence, throughout the paper we will only discuss the results obtained with the parametrization D1M.

In Sec. II we give a brief account of the method used to describe the considered odd-mass nuclei, and then present the parameters for the boson-core Hamiltonian, single-particle energies and occupation probabilities for the odd particle, and the fitted strength parameters for the boson-fermion coupling interaction. In Sec. III, we present the results for the even-even core nuclei, including the Gogny-D1M and mapped energy surfaces, and the calculated low-energy excitation spectra in comparison to the experimental data. In Sec. IV, the results for the spectroscopic calculation for the odd-mass $\mathrm{Eu}$ and $\mathrm{Sm}$ isotopes, including evolution of energy levels, $B(E 2)$, and spectroscopic quadrupole and magnetic moments, are discussed. In Sec. V the energy spectra and decay patterns in the $\gamma$-soft nuclei ${ }^{195} \mathrm{Pt}$ and ${ }^{195} \mathrm{Au}$ are discussed and compared with the available experimental data. Finally, Sec. VI is devoted to the summary of the paper and to discussing future perspectives.

\section{DESCRIPTION OF THE MODEL}

In this section, we briefly describe the theoretical framework proposed in Ref. [21] and used in this study. We also discuss the parameters of the IBFM Hamiltonian employed in the calculations. For more details on the philosophy of the model as well as its main assumptions the reader is referred to Ref. [21] for a thorough discussion.

\section{A. Construction of IBFM Hamiltonian}

The IBFM Hamiltonian, used to describe the studied oddmass nuclei, consists of three terms, namely, the even-even boson core or interacting boson model (IBM) Hamiltonian $\hat{H}_{B}$, the single-particle Hamiltonian for unpaired fermions $\hat{H}_{F}$, and the boson-fermion coupling term $\hat{H}_{B F}$ :

$$
\hat{H}=\hat{H}_{B}+\hat{H}_{F}+\hat{H}_{B F} .
$$

The building blocks of the IBM are the traditional $s$ and $d$ bosons, which represent the collective pairs of valence nucleons [27] coupled to angular momenta $J^{\pi}=0^{+}$and $2^{+}$, respectively. The numbers of bosons $N_{B}$ and fermions $N_{F}$ are assumed to be conserved separately. Note also that we use the traditional version of the IBM where there is no distinction between neutron and proton bosons. Finally, we assume that, because we only consider odd-mass nuclei, the number of fermions equals one $N_{F}=1$. The IBM Hamiltonian $\hat{H}_{B}$ reads

$$
\hat{H}_{B}=\epsilon_{d} \hat{n}_{d}+\kappa \hat{Q}_{B} \cdot \hat{Q}_{B}+\kappa^{\prime} \hat{L} \cdot \hat{L},
$$

given in terms of the $d$-boson number operator $\hat{n}_{d}=d^{\dagger} \cdot \tilde{d}$, the quadrupole operator $\hat{Q}_{B}=s^{\dagger} \tilde{d}+d^{\dagger} \tilde{s}+\chi\left[d^{\dagger} \times \tilde{d}\right]^{(2)}$, and the angular momentum operator $\hat{L}=\sqrt{10}\left[d^{\dagger} \times \tilde{d}\right]^{(1)}$. The remaining quantities $\epsilon_{d}, \kappa, \kappa^{\prime}$, and $\chi$ are parameters of the Hamiltonian $\hat{H}_{B}$.

On the other hand, the single-fermion Hamiltonian takes the form

$$
\hat{H}_{F}=\sum_{j} \epsilon_{j}\left[a_{j}^{\dagger} \times \tilde{a}_{j}\right]^{(0)}
$$

where $a_{j}^{\dagger}$ and $a_{j}$ are the fermion creation and annihilation operators while $\epsilon_{j}$ stands for the single-particle energy of the orbital $j$.

For the boson-fermion coupling Hamiltonian $\hat{H}_{B F}$ we have employed the simplest possible form, as suggested in Refs. [16,17]:

$$
\begin{aligned}
\hat{H}_{B F}= & \sum_{j j^{\prime}} \Gamma_{j j^{\prime}} \hat{Q}_{B} \cdot\left[a_{j}^{\dagger} \times \tilde{a}_{j^{\prime}}\right]^{(2)} \\
& +\sum_{j j^{\prime} j^{\prime \prime}} \Lambda_{j j^{\prime}}^{j^{\prime \prime}}:\left[\left[d^{\dagger} \times \tilde{a}_{j}\right]^{\left(j^{\prime \prime}\right)} \times\left[a_{j^{\prime}}^{\dagger} \times \tilde{d}\right]^{\left(j^{\prime \prime}\right)}\right]^{(0)}: \\
& +\sum_{j} A_{j}\left[a^{\dagger} \times \tilde{a}_{j}\right]^{(0)} \hat{n}_{d},
\end{aligned}
$$

where the first, second, and third terms are referred to as the quadrupole, exchange, and monopole interactions, 
respectively. The strength parameters $\Gamma_{j j^{\prime}}, \Lambda_{j j^{\prime}}^{j^{\prime \prime}}$, and $A_{j}$ can be expressed, using the generalized seniority scheme, in the following $j$-dependent forms [16]:

$$
\begin{aligned}
\Gamma_{j j^{\prime}} & =\Gamma_{0} \gamma_{j j^{\prime}}, \\
\Lambda_{j j^{\prime}}^{j^{\prime \prime}} & =-2 \Lambda_{0} \sqrt{\frac{5}{2 j^{\prime \prime}+1}} \beta_{j j^{\prime \prime}} \beta_{j^{\prime} j^{\prime \prime}}, \\
A_{j} & =-A_{0} \sqrt{2 j+1},
\end{aligned}
$$

where $\quad \gamma_{j j^{\prime}}=\left(u_{j} u_{j^{\prime}}-v_{j} v_{j^{\prime}}\right) Q_{j j^{\prime}} \quad$ and $\quad \beta_{j j^{\prime}}=\left(u_{j} v_{j^{\prime}}+\right.$ $\left.v_{j} u_{j^{\prime}}\right) Q_{j j^{\prime}}$, with the matrix element of the quadrupole operator in the single-particle basis $Q_{j j^{\prime}}=\left\langle j\left\|Y^{(2)}\right\| j^{\prime}\right\rangle$. Both $u_{j}$ and $v_{j}$ represent the occupation probabilities for the orbital $j$ and satisfy the well known relation $u_{j}^{2}+v_{j}^{2}=1$. Furthermore, $\Gamma_{0}, \Lambda_{0}$, and $A_{0}$ denote the corresponding strength parameters. Note that an exhaustive presentation of the physical contents of the formulas in Eqs. (4)-(7) as well as the discussion of relevant applications to odd- $A$ nuclei, were already considered in Ref. [16].

The first step to build the IBFM Hamiltonian $\hat{H}$ in Eq. (1) is to determine the IBM Hamiltonian $\hat{H}_{B}$ by using the fermionto-boson mapping procedure developed in Refs. [28-30]. Here, the fermion $(\beta \gamma)$-deformation energy surface, obtained within the constrained Gogny-D1M HFB framework, is mapped onto the expectation value of $\hat{H}_{B}$ in the boson condensate state [31]. This procedure completely determines the parameters $\epsilon_{d}, \kappa$, and $\chi$. The strength parameter $\kappa^{\prime}$ for the $\hat{L} \cdot \hat{L}$ term is obtained separately by equating the cranking moment of inertia, calculated in the boson coherent state at the energy minimum, to the corresponding Thouless-Valatin moment of inertia computed within the cranked HFB approach [30]. It was shown in Ref. [30] that the $\hat{L} \cdot \hat{L}$ term is only relevant in axially deformed systems and, for that reason, we do not included it in the calculation for the $\gamma$-soft nucleus ${ }^{194} \mathrm{Pt}$. For a more detailed account of constrained Gogny-HFB calculations the reader is referred, for example, to Refs. [32,33]. Details of the fermion-to-boson mapping procedure are given in Refs. [28,29]. The parameters derived for the isotopes ${ }^{148-154} \mathrm{Sm}$ and ${ }^{194} \mathrm{Pt}$ can be found in Table I.

For the fermion valence space, we have included all the spherical single-particle orbitals in the proton major shell $Z=$ 50-82 (i.e., $3 s_{1 / 2}, 2 d_{3 / 2}, 2 d_{5 / 2}$, and $1 g_{7 / 2}$ for positive-parity states and $1 h_{11 / 2}$ for the intruder negative-parity states) for the odd-mass systems ${ }^{149-155} \mathrm{Eu}$ and ${ }^{195} \mathrm{Au}$.

On the other hand, in the case of ${ }^{149-155} \mathrm{Sm}$ and ${ }^{195} \mathrm{Pt}$, with the Fermi level lying in the neutron major shell $N=82-126$,

TABLE I. Parameters $\left(\epsilon_{d}, \kappa, \kappa^{\prime}\right.$, and $\left.\chi\right)$ of the boson Hamiltonian $\hat{H}_{B}$. All entries, except the dimensionless parameter $\chi$, are in $\mathrm{MeV}$.

\begin{tabular}{lcccr}
\hline \hline & $\epsilon_{d}$ & $\kappa$ & $\kappa^{\prime}$ & $\chi$ \\
\hline${ }^{148} \mathrm{Sm}$ & 1.185 & -0.079 & -0.027 & -0.44 \\
${ }^{150} \mathrm{Sm}$ & 0.615 & -0.074 & -0.014 & -0.50 \\
${ }^{152} \mathrm{Sm}$ & 0.336 & -0.074 & -0.018 & -0.50 \\
${ }^{154} \mathrm{Sm}$ & 0.106 & -0.074 & -0.018 & -0.50 \\
${ }^{194} \mathrm{Pt}$ & 0.011 & -0.098 & & 0.10 \\
\hline \hline
\end{tabular}

TABLE II. Spherical single-particle energies (in MeV) resulting from Gogny-D1M HFB calculations for the considered odd-mass nuclei. For details, see the main text.

\begin{tabular}{lllllll}
\hline \hline & $3 s_{1 / 2}$ & $2 d_{3 / 2}$ & $2 d_{5 / 2}$ & $1 g_{7 / 2}$ & $1 h_{11 / 2}$ & \\
\hline${ }^{149} \mathrm{Eu}$ & 3.365 & 3.076 & 0.868 & 0.000 & 3.512 & \\
${ }^{151} \mathrm{Eu}$ & 3.378 & 3.063 & 0.850 & 0.000 & 3.544 & \\
${ }^{153} \mathrm{Eu}$ & 3.425 & 3.078 & 0.876 & 0.000 & 3.593 & \\
${ }^{155} \mathrm{Eu}$ & 3.494 & 3.114 & 0.936 & 0.000 & 3.653 & \\
${ }^{195} \mathrm{Au}$ & 0.000 & 0.907 & 2.624 & 5.164 & 0.840 & \\
& $3 p_{1 / 2}$ & $3 p_{3 / 2}$ & $2 f_{5 / 2}$ & $2 f_{7 / 2}$ & $1 h_{9 / 2}$ & $1 i_{13 / 2}$ \\
${ }^{149} \mathrm{Sm}$ & 3.528 & 2.607 & 3.049 & 0.000 & 1.191 & 3.310 \\
${ }^{151} \mathrm{Sm}$ & 3.491 & 2.573 & 3.052 & 0.000 & 1.141 & 3.268 \\
${ }^{153} \mathrm{Sm}$ & 3.458 & 2.544 & 3.041 & 0.000 & 1.076 & 3.214 \\
${ }^{155} \mathrm{Sm}$ & 3.430 & 2.521 & 3.021 & 0.000 & 1.005 & 3.154 \\
${ }^{195} \mathrm{Pt}$ & 0.000 & 0.927 & 1.014 & 3.816 & 4.273 & 1.495 \\
\hline \hline
\end{tabular}

we have considered the positive parity intruder orbital $1 i_{13 / 2}$ and the negative parity orbitals $3 p_{1 / 2}, 3 p_{3 / 2}, 2 f_{5 / 2}, 2 f_{7 / 2}$, and $1 h_{9 / 2}$.

Following the prescription of Ref. [21], the single-particle energies $\epsilon_{j}$ and the occupation probabilities $v_{j}^{2}$ are obtained from self-consistent Gogny-D1M HFB calculations for the spherical configuration. In those calculations, for a given odd-mass nucleus with the odd neutron (proton) number $N_{0}$ $\left(Z_{0}\right)$, the standard even number parity constrained GognyHFB approach (i.e., without blocking) was used, but using $N_{0}\left(Z_{0}\right)$ for the neutron (proton) number constraint. The single-particle energies and occupation probabilities obtained for the considered odd- $A$ nuclei are shown in Tables II and III, respectively.

The coupling constants of the boson-fermion interaction term $\hat{H}_{B F}\left(\Gamma_{0}, \Lambda_{0}\right.$, and $\left.A_{0}\right)$ are the only free parameters in our study. They are fitted, for each nucleus, to reproduce the lowest few experimental energy levels, separately for positiveand negative-parity states [21]. We show in Table IV the fitted strength parameters for the positive- $\left(\Gamma_{0}^{+}, \Lambda_{0}^{+}\right.$, and $\left.A_{0}^{+}\right)$and negative-parity $\left(\Gamma_{0}^{-}, \Lambda_{0}^{-}\right.$, and $\left.A_{0}^{-}\right)$states.

TABLE III. Occupation probabilities of the single-particle orbitals resulting from Gogny-D1M HFB calculations for the considered odd-mass nuclei. For details, see the main text.

\begin{tabular}{lllllll}
\hline \hline & $3 s_{1 / 2}$ & $2 d_{3 / 2}$ & $2 d_{5 / 2}$ & $1 g_{7 / 2}$ & $1 h_{11 / 2}$ & \\
\hline${ }^{149} \mathrm{Eu}$ & 0.112 & 0.158 & 0.700 & 0.843 & 0.102 & \\
${ }^{151} \mathrm{Eu}$ & 0.110 & 0.159 & 0.705 & 0.845 & 0.099 & \\
${ }^{153} \mathrm{Eu}$ & 0.107 & 0.159 & 0.706 & 0.851 & 0.095 & \\
${ }^{155} \mathrm{Eu}$ & 0.104 & 0.159 & 0.703 & 0.858 & 0.092 & \\
${ }^{195} \mathrm{Au}$ & 0.617 & 0.870 & 0.968 & 0.989 & 0.864 & \\
& $3 p_{1 / 2}$ & $3 p_{3 / 2}$ & $2 f_{5 / 2}$ & $2 f_{7 / 2}$ & $1 h_{9 / 2}$ & $1 i_{13 / 2}$ \\
${ }^{149} \mathrm{Sm}$ & 0.013 & 0.023 & 0.027 & 0.413 & 0.126 & 0.022 \\
${ }^{151} \mathrm{Sm}$ & 0.020 & 0.036 & 0.039 & 0.531 & 0.202 & 0.034 \\
${ }^{153} \mathrm{Sm}$ & 0.028 & 0.053 & 0.053 & 0.623 & 0.291 & 0.049 \\
${ }^{155} \mathrm{Sm}$ & 0.038 & 0.075 & 0.069 & 0.693 & 0.387 & 0.067 \\
${ }^{195} \mathrm{Pt}$ & 0.303 & 0.603 & 0.634 & 0.956 & 0.961 & 0.763 \\
\hline \hline
\end{tabular}


TABLE IV. Fitted parameters of the boson-fermion Hamiltonian $\hat{H}_{B F}\left(\Gamma_{0}^{ \pm}, \Lambda_{0}^{ \pm}\right.$, and $\left.A_{0}^{ \pm}\right)$. All entries are in $\mathrm{MeV}$.

\begin{tabular}{lccclll}
\hline \hline & $\Gamma_{0}^{+}$ & $\Lambda_{0}^{+}$ & $A_{0}^{+}$ & $\Gamma_{0}^{-}$ & $\Lambda_{0}^{-}$ & $A_{0}^{-}$ \\
\hline${ }^{149} \mathrm{Eu}$ & 0.05 & 2.5 & -0.13 & 0.3 & 3.5 & -0.14 \\
${ }^{151} \mathrm{Eu}$ & 0.06 & 1.0 & 0.0 & 0.6 & 6.5 & -0.06 \\
${ }^{153} \mathrm{Eu}$ & 0.17 & 7.0 & -0.65 & 0.6 & 9.0 & -0.30 \\
${ }^{155} \mathrm{Eu}$ & 0.19 & 4.5 & -0.44 & 0.5 & 8.0 & -0.30 \\
${ }^{149} \mathrm{Sm}$ & 0.2 & 36.0 & -0.25 & 0.2 & 1.05 & -0.15 \\
${ }^{151} \mathrm{Sm}$ & 1.4 & 39.0 & -0.30 & 0.3 & 0.15 & -0.18 \\
${ }^{153} \mathrm{Sm}$ & 1.9 & 35.0 & -0.18 & 0.7 & 2.5 & -0.50 \\
${ }^{155} \mathrm{Sm}$ & 1.6 & 22.5 & -0.28 & 0.58 & 1.25 & -0.30 \\
${ }^{195} \mathrm{Pt}$ & 0.5 & 0.6 & -0.36 & 0.6 & 0.5 & -0.85 \\
${ }^{195} \mathrm{Au}$ & 0.6 & 1.45 & -0.35 & 0.65 & 2.0 & -0.33 \\
\hline \hline
\end{tabular}

Once all the parameters of the different building blocks of the IBFM Hamiltonian $\hat{H}$ are fixed by the procedure described above, the Hamiltonian is diagonalized in the spherical basis $|j, L, \alpha, J\rangle$ [34], where $\alpha=\left(n_{d}, v, n_{\Delta}\right)$ represents a generic notation for the quantum numbers of the $\mathrm{U}(5)$ symmetry in the IBM [35], and $L$ and $J$ are the angular momentum of the boson and the total angular momentum of the coupled boson-fermion system, respectively. They satisfy the standard triangular selection rule $|L-j| \leqslant J \leqslant L+j$. The wave functions resulting from the diagonalization of $\hat{H}$ are used to compute the $B(E 2)$ and $B(M 1)$ transition rates as well as spectroscopic quadrupole and magnetic moments. The electric $E 2$ transition operator is taken as the sum of the boson and fermion parts

$$
\hat{T}^{(E 2)}=\hat{T}_{B}^{(E 2)}+\hat{T}_{F}^{(E 2)}
$$

with the boson operator given by

$$
T_{B}^{(E 2)}=e_{B} \hat{Q}_{B},
$$

where $e_{B}$ is the boson effective charge and $\hat{Q}_{B}$ represents the quadrupole operator defined in Eq. (2) with the same value of the parameter $\chi$. On the other hand, the fermion $E 2$ operator takes the form

$$
\hat{T}_{F}^{(E 2)}=-e_{F} \sum_{j j^{\prime}} \frac{1}{\sqrt{5}} \gamma_{j j^{\prime}}\left[a^{\dagger} \times \tilde{a}_{j^{\prime}}\right]^{(2)},
$$

with $e_{F}$ being the fermion effective charge. As in previous studies [21,23], $e_{B}$ is fitted to reproduce the experimental $B\left(E 2 ; 2_{1}^{+} \rightarrow 0_{1}^{+}\right)$value of the corresponding even-even bosoncore nuclei while $e_{F}$ is taken as $e_{F}=e_{B}$ for all the considered odd-mass nuclei.

In the same fashion, the magnetic $M 1$ transition operator is given by

$$
\hat{T}^{(M 1)}=\sqrt{\frac{3}{4 \pi}}\left(\hat{T}_{B}^{(M 1)}+\hat{T}_{F}^{(M 1)}\right)
$$

where the M1 boson operator is proportional to the boson angular momentum operator $\hat{T}_{B}^{(M 1)}=g_{B} \hat{L}$ with the gyromagnetic factor $g_{B}=\mu_{2_{1}^{+}} / 2$ given in terms of the magnetic moment $\mu_{2_{1}^{+}}$ of the $2_{1}^{+}$state of the even-even nucleus. The corresponding experimental value is used for this quantity. The fermion part is written as $\hat{T}_{F}^{(M 1)}$ and takes the form [16]

$$
\hat{T}_{F}^{(M 1)}=-\sum_{j j^{\prime}} g_{j j^{\prime}} \sqrt{\frac{j(j+1)(2 j+1)}{3}}\left[a_{j}^{\dagger} \times \tilde{a}_{j^{\prime}}\right]^{(1)},
$$

with the coefficients $g_{j j^{\prime}}$ given by

$$
g_{j j^{\prime}}= \begin{cases}\frac{(2 j-1) g_{l}+g_{s}}{2 j} & \left(j=j^{\prime}=l+\frac{1}{2}\right), \\ \frac{(2 j+3) g_{l}-g_{s}}{2(j+1)} & \left(j=j^{\prime}=l-\frac{1}{2}\right), \\ \left(g_{l}-g_{s}\right) \sqrt{c \frac{2 l(l+1)}{j(j+1)(2 j+1)(2 l+1)}} & \left(j^{\prime}=j-1 ; l=l^{\prime}\right) .\end{cases}
$$

In this expression $l$ represents the orbital angular momentum of the single-particle state. Throughout this work, the fermion $g_{l}$ and $g_{s}$ gyromagnetic factors take the usual Schmidt values $g_{l}=1.0 \mu_{N}^{2}$ and $g_{s}=5.58 \mu_{N}^{2}$ for the proton and $g_{l}=0$ and $g_{s}=-3.82 \mu_{N}^{2}$ for the neutron. The $g_{s}$ is quenched by $30 \%$ for both proton and neutron, as in Refs. [21,36].

\section{B. Comparison of the parameters with the ones of the relativistic EDF}

At this point, it is worthwhile to point out some differences found between the values of the parameters entering the IBFM Hamiltonian, especially $\hat{H}_{F}$ and $\hat{H}_{B F}$, obtained for the odd- $A$ $\mathrm{Eu}$ and $\mathrm{Sm}$ isotopes in the present work as compared to the ones used in the previous studies of the same isotopes within the relativistic EDF framework [21,23].

First, in Figs. 1 and 2, we plot the strength parameters $\Gamma_{0}^{ \pm}, \Lambda_{0}^{ \pm}$, and $A_{0}^{ \pm}$obtained for the odd- $A$ Eu and Sm isotopes, respectively, from the Gogny-D1M and relativistic DD-PC1 EDFs. As one can observe in Figs. 1(a) and 1(b), significant discrepancies between the present and previous $[21,23]$ studies are found in the fitted $\Lambda_{0}^{ \pm}$and $A_{0}^{ \pm}$values, especially for lighter isotopes ${ }^{149,151} \mathrm{Eu}$, both quantitatively and qualitatively. In addition, in Ref. [21] the monopole term was introduced only for the $2 d_{5 / 2}$ orbit for the positive-parity states in the odd-mass Eu isotopes. In this study, on the one hand, we have introduced the monopole term for all the positive-parity orbitals in a given isotope, with a common value $A_{0}^{+}$. On the other hand, the values of the strength parameters $\left(\Gamma_{0}^{ \pm}, \Lambda_{0}^{ \pm}\right.$, and $\left.A_{0}^{ \pm}\right)$obtained here for the odd- $A$ Sm isotopes (see Fig. 2) are quite similar to those employed in Ref. [23], except perhaps for the $A_{0}^{+}$values for ${ }^{149} \mathrm{Sm}$ [see Fig. 2(c)].

The observed differences in the boson-fermion strength parameters for the odd- $A$ Eu between the present and previous [21] studies could partly originate from the quantitative differences, especially in the single-particle energies, between the Gogny and relativistic EDF quantities entering the fit. To illustrate this possibility, we plot in Fig. 3 the spherical single-particle energies and occupation probabilities for the odd- $A$ Eu isotopes obtained in Ref. [21] and in the present work. As seen in panels (a) and (b) of Fig. 3, the energy gap between the $1 g_{7 / 2}$ and $2 d_{5 / 2}$ orbitals for the odd-mass Eu isotopes is, in general, more than $3 \mathrm{MeV}$ in Ref. [21] while it is less than $1 \mathrm{MeV}$ in the Gogny-D1M calculations (see also 


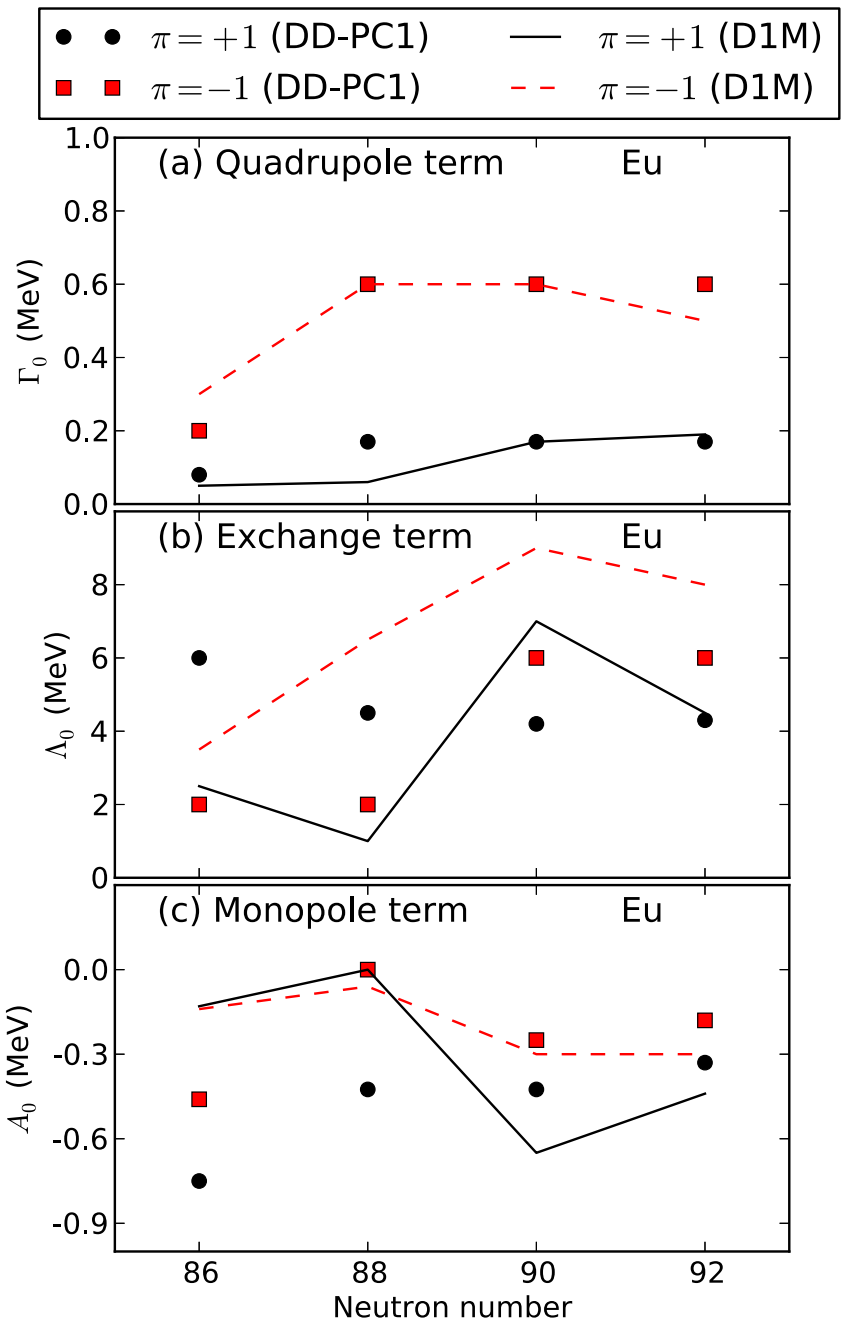

FIG. 1. The coupling constants of the boson-fermion interaction term $\hat{H}_{B F}$ for the odd- $A$ Eu isotopes, used in the present study (denoted by "D1M") and in the previous calculation in Refs. [21,23]. They are shown separately for positive- $(\pi=+1)$ and negative-parity $(\pi=-1)$ states.

Table II). The values of $\epsilon_{j}$ and $v_{j}^{2}$ obtained for the odd- $A$ Sm isotopes from the Gogny-D1M and the relativistic EDFs are plotted in Fig. 4.

In addition, we observe in Fig. 2(b) as well as in Table IV that the values of $\Lambda_{0}^{+}$for ${ }^{149-155} \mathrm{Sm}$ are rather large $(\approx 20-40$ $\mathrm{MeV})$, about a factor of 10 larger than the $\Lambda_{0}^{ \pm}$parameters obtained for other odd-mass nuclei. They are also larger than the ones employed in earlier phenomenological IBFM calculations for other isotopic chains [18]. The reason for the large $\Lambda_{0}^{+}$values in the odd-mass Sm nuclei is the nearly vanishing $\beta_{j j^{\prime \prime}} \beta_{j^{\prime} j^{\prime \prime}}$ factors in the strength parameters $\Lambda_{j j^{\prime}}^{j^{\prime \prime}}$ [see Eq. (6)]; a consequence of the too small $v_{i_{13 / 2}}^{2}$ values used [see Table III and Fig. 4(d)]. We note that even larger values of $\Lambda_{0}^{+}$for the odd- $A$ Sm isotopes than the present ones were obtained in the case where the relativistic DD-PC1 EDF was used [23] [see Fig. 2(b)]. In Ref. [23], too small $v_{i_{13 / 2}}^{2}$ values for the odd- $A \mathrm{Sm}$ isotopes were also obtained, similarly to the present work [see Fig. 4(d)].

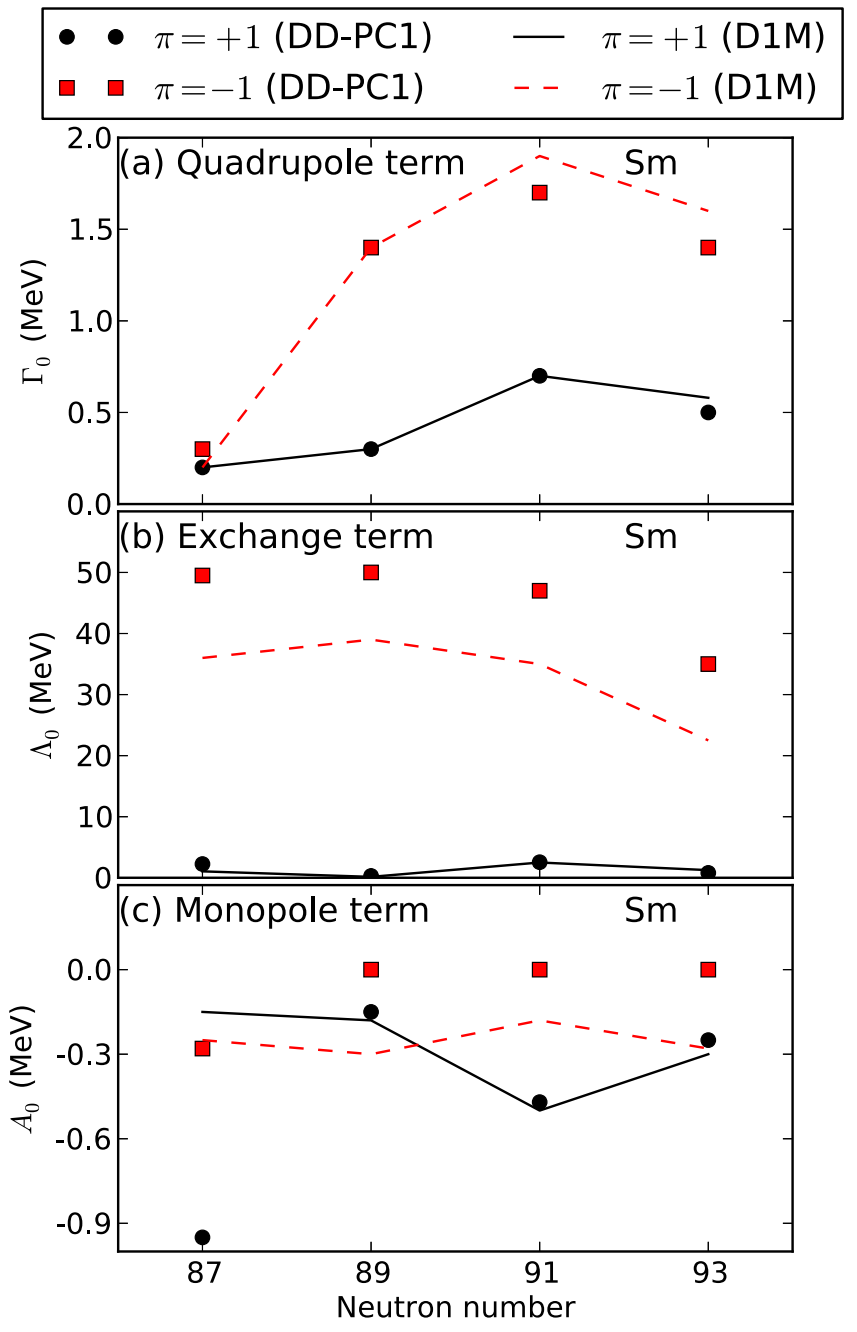

FIG. 2. The same as in Fig. 1, but for the odd- $A$ Sm isotopes.

\section{RESULTS FOR THE EVEN-EVEN CORE NUCLEI}

The Gogny-D1M and mapped IBM energy surfaces obtained for the even-even boson-core nuclei ${ }^{148-154} \mathrm{Sm}$ are plotted in Fig. 5. Those surfaces illustrate the transition between nearly spherical and axially deformed shapes [38]. In the case of ${ }^{148} \mathrm{Sm}$ the HFB surface exhibits a weakly deformed minimum around $\beta=0.15$. The nucleus ${ }^{150} \mathrm{Sm}$ displays a sharper potential in both $\beta$ and $\gamma$ directions with a minimum around $\beta=0.2$. The minimum of the HFB surface obtained for ${ }^{152} \mathrm{Sm}$ is even sharper, especially along the $\gamma$ direction, but it looks softer in $\beta$ than in ${ }^{150} \mathrm{Sm}$. This softening of the potential in $\beta$ agrees well with a key feature of a transitional nucleus associated with the $\mathrm{X}(5)$ critical-point symmetry $[39,40]$. Finally, the nucleus ${ }^{154} \mathrm{Sm}$ exhibits the most pronounced prolate deformation with $\beta \approx 0.35$. The mapped IBM surfaces, plotted in the lower row of the figure, reproduce nicely the Gogny-HFB ones for each nucleus, except that, due to the limited IBM configuration space used in this work, far away from the minimum the IBM surfaces tend to be flatter than the Gogny-HFB ones. In Fig. 6, we have plotted the Gogny-D1M and IBM energy contour plots for ${ }^{194} \mathrm{Pt}$. Both 


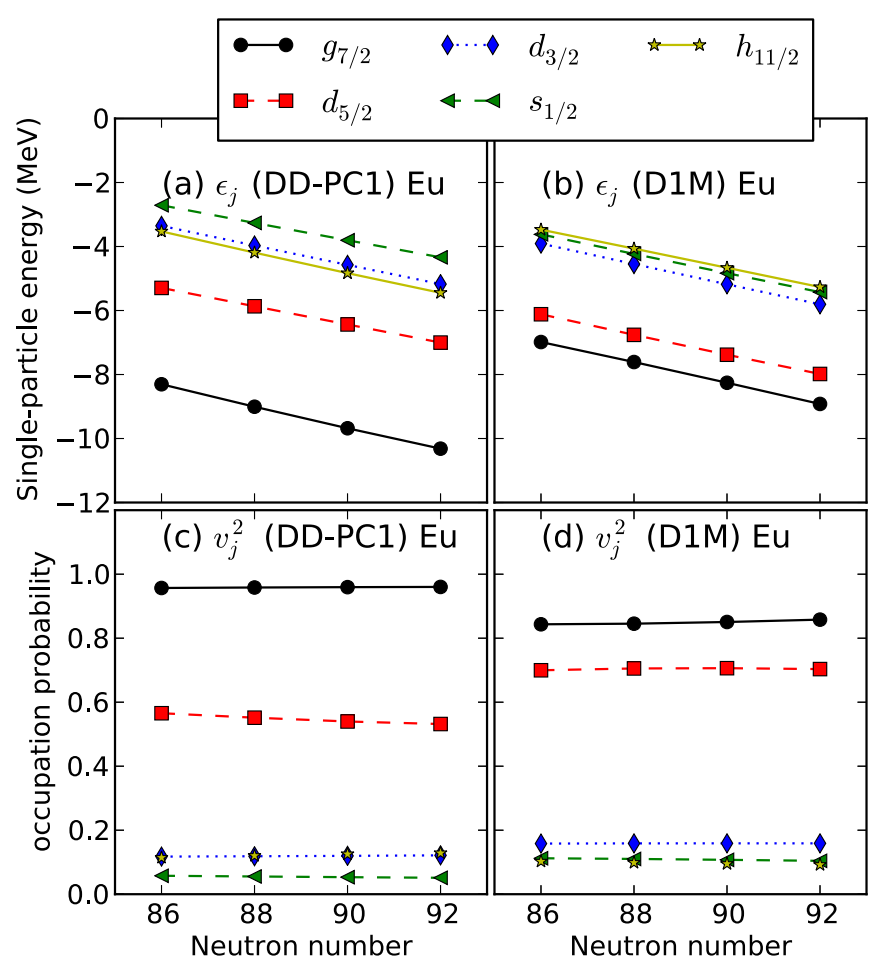

FIG. 3. The spherical single-particle energies $\epsilon_{j}$ and occupation probabilities $v_{j}^{2}$ for the odd- $A$ Eu isotopes, resulting from the GognyD1M HFB calculations, are compared with those obtained with the relativistic DD-PC1 EDF in Ref. [23].

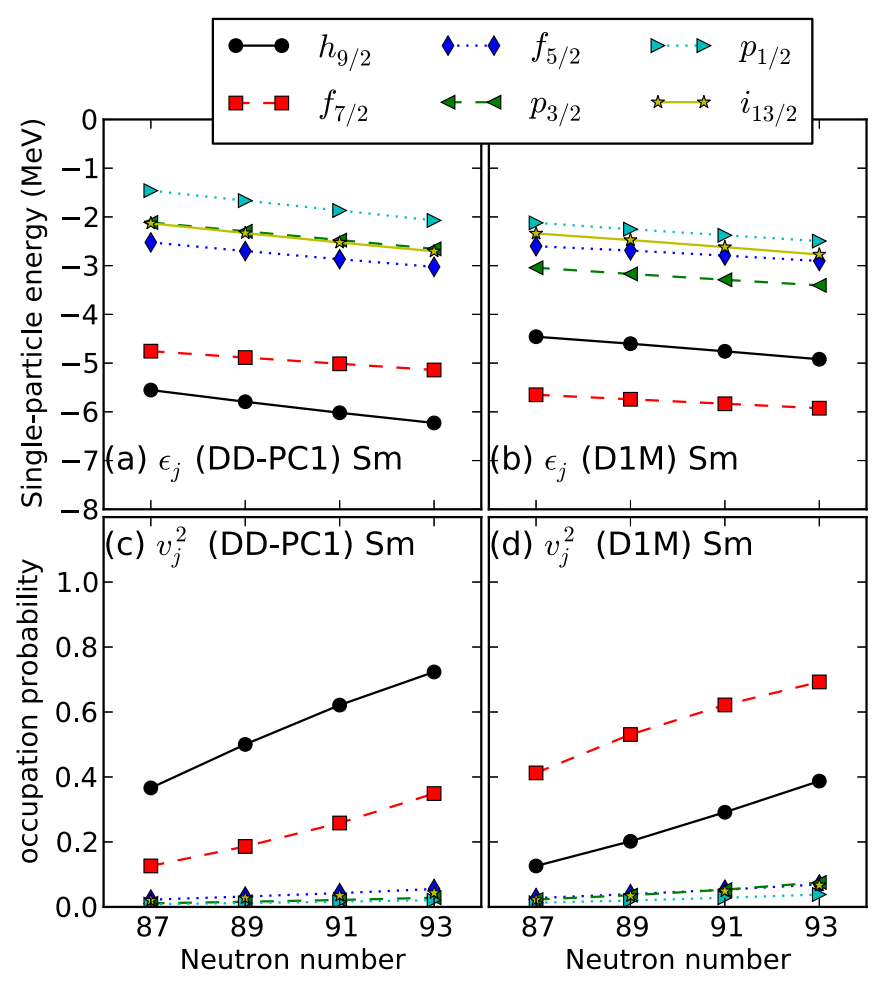

FIG. 4. The same as Fig. 3, but for the odd- $A$ Sm isotopes. surfaces exhibit a typical $\gamma$ softness with a weakly deformed oblate minimum at $\beta \approx 0.15$

The low-energy levels, resulting from the diagonalization of the IBM Hamiltonian, are plotted in Fig. 7 as functions of the neutron number $N$ for the isotopes ${ }^{148-154} \mathrm{Sm}$. They are compared with the available experimental data [37]. The calculations reproduce reasonably well the experimental trends of the low-lying energy levels and suggest the transition from a vibrational-like spectrum at $N=86$ to the typical rotational-like spectrum at $N=92$. The overestimation of the energies of the $0_{2}^{+}$and $2_{2}^{+}$states was also found in earlier calculations within the fermion-to-boson mapping procedure $[28,29]$. A reason for that could be the restricted model space of the IBM and/or to the fact that the shape of the Gogny-HFB energy surfaces around the minimum have too large curvatures in both the $\beta$ and $\gamma$ directions, which requires a large value of the quadrupole-quadrupole interaction strength $\kappa$ [see Eq. (2)] in the IBM Hamiltonian. The large $\kappa$ values push up the non-yrast energy levels.

The low-energy spectrum obtained for ${ }^{194} \mathrm{Pt}$ is shown in Fig. 8. It exhibits several features of $\gamma$ softness or the $\mathrm{O}(6)$ symmetry [35], i.e., the energy ratio $R_{4 / 2}=E\left(4_{1}^{+}\right) / E\left(2_{1}^{+}\right)=2.57$, the multiplets $\left(4_{1}^{+}, 2_{2}^{+}\right)$and $\left(6_{1}^{+}, 4_{2}^{+}, 3_{1}^{+}\right)$, the large $B\left(E 2 ; 2_{2}^{+} \rightarrow\right.$ $\left.2_{1}^{+}\right)$transition strength of the same order of magnitude as the $B\left(E 2 ; 4_{1}^{+} \rightarrow 2_{1}^{+}\right)$one, and the selection rule of the $E 2$ decay from the $0_{2}^{+}$to $2_{1,2}^{+}$state. When compared with the experimental data, the spectrum looks rather stretched.

\section{SPECTROSCOPIC PROPERTIES OF ODD- $A$ Eu AND Sm NUCLEI}

Having demonstrated that the mapped IBM Hamiltonian gives a reasonable description of the even-even (boson-core) nuclei, we now turn our attention to the considered odd-mass nuclei. In Fig. 9 we have depicted the low-lying positiveand negative-parity states obtained for the studied odd- $A \mathrm{Eu}$ isotopes as functions of neutron number $N$. They are compared with the available experimental data [37]. A reasonable good agreement between the theoretical predictions and the experimental values is observed. The compression of the positive- and negative-parity levels, as functions of the neutron number, correlates well with the lowering of the yrast levels in the even-even nuclei (see Fig. 7). It can be regarded as a signature of the structural evolution from the nearly spherical to the well-deformed regime. Another signature of shape transition, specific to the odd-mass nuclei, is the change in the angular momentum of the ground state in the case of negative-parity configurations from $N=88$ to 90 [see panels (c) and (d)]. Our results suggest that for ${ }^{149,151} \mathrm{Eu}$ the $11 / 2_{1}^{-}$is the ground state for negative parity, which is weakly coupled to the boson core (represented by ${ }^{148,150} \mathrm{Sm}$ ) that exhibits a moderate deformation (see Fig. 5). At $N=90$ and 92, the coupling between the odd proton and boson-core nucleus becomes stronger and the regular rotational band built on the $5 / 2_{1}^{-}$state, that follows the $\Delta J=1$ systematics of the strong coupling limit, emerges. In the case of the positive-parity states [panels (a) and (b)], the $5 / 2^{+}$one remains the ground state. Nevertheless, both theoretically and experimentally, the $5 / 2_{1}^{+}$ and $7 / 2_{1}^{+}$states are rather close in energy at $N=88$. This 


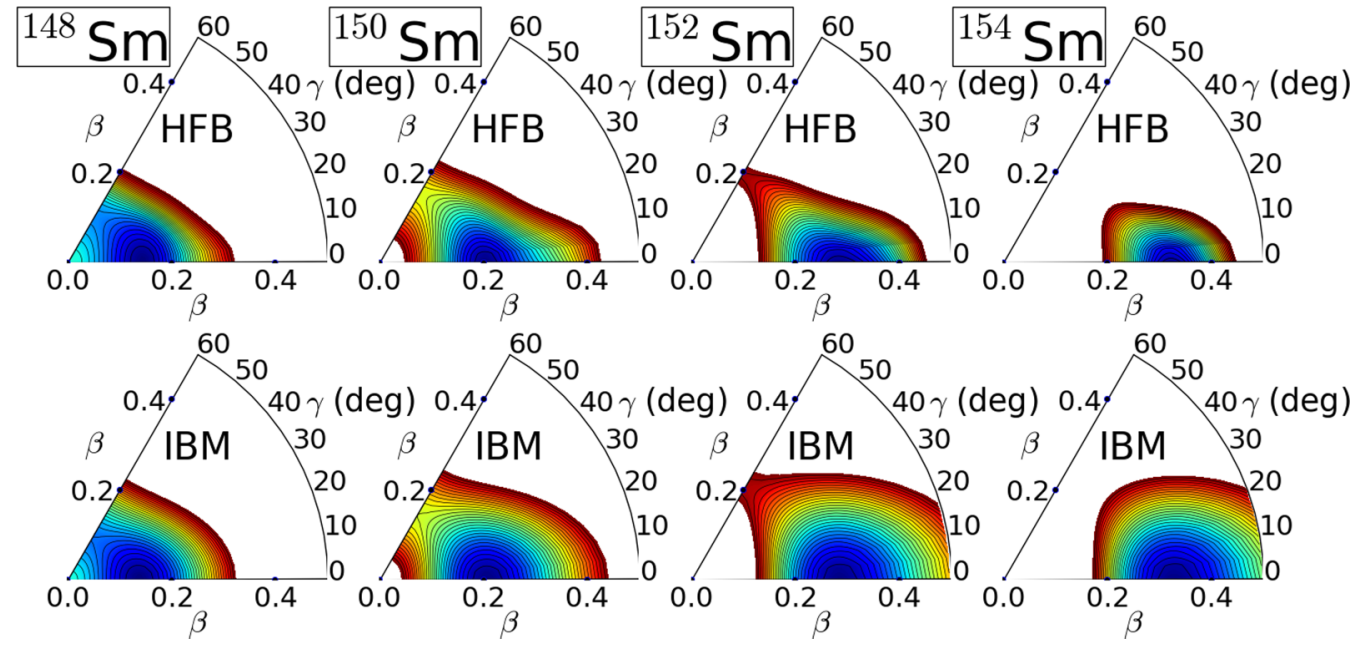

FIG. 5. The Gogny-D1M (upper row) and mapped IBM (lower row) energy surfaces for the even-even nuclei ${ }^{148-154}$ Sm are plotted up to $3 \mathrm{MeV}$ above the absolute minimum. The difference between neighboring contours is $100 \mathrm{keV}$.

feature can be regarded as a signature of the structural change taking place around $N=88$.

The theoretical and experimental spectra for the odd-mass $\mathrm{Sm}$ isotopes are plotted in Fig. 10. As can be seen, our calculations provide a reasonable description of the experimental data. A change in the spin of the ground state is observed at $N=91$. On the other hand, the levels are more compressed from $N=87$ towards 89 or 91 [panels (a), (c), and (d)] suggesting a structural change in those odd-mass systems. The experimental data indicate that for the transitional isotope ${ }^{153} \mathrm{Sm}$ many levels are found below $\approx 300 \mathrm{keV}$. They also reveal [panels (b) and (d)] a more regular rotational-like band that exhibits the $\Delta J=1$ systematics of the strong coupling regime. Our results suggest that for both parities the rotational-like band appears already at $N=91$ [panels (a) and (c)]. However, the predicted negative-parity levels for the $N=91$ and 93 nuclei [see panel (c)], look rather irregular, with the staggering pattern $\left(3 / 2_{1}^{-}, 5 / 2_{1}^{-}\right),\left(7 / 2_{1}^{-}, 9 / 2_{1}^{-}\right),\left(11 / 2_{1}^{-}, 13 / 2_{1}^{-}\right), \ldots$. We notice here that the Gogny-D1M energy systematic obtained for the odd-mass Eu and Sm nuclei is similar to the results found within the relativistic EDF framework [21,23].

In addition to the energy spectra, the electromagnetic transition rates also provide signatures of the structural evolution in the considered odd-mass nuclei. The $B\left(E 2 ; 9 / 2_{1}^{+} \rightarrow 5 / 2_{1}^{+}\right)$, $B\left(E 2 ; 7 / 2_{1}^{+} \rightarrow 5 / 2_{1}^{+}\right)$, and $B\left(E 2 ; 5 / 2_{2}^{+} \rightarrow 7 / 2_{1}^{+}\right)$transition

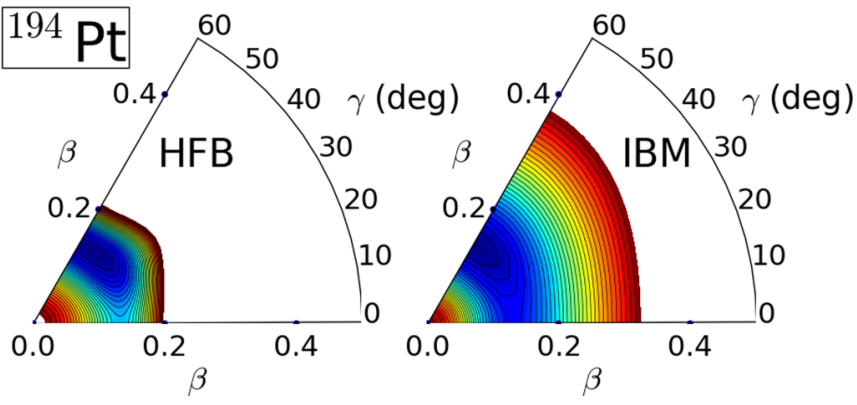

FIG. 6. Same as in Fig. 5, but for ${ }^{194} \mathrm{Pt}$. probabilities obtained for the odd-mass Eu isotopes are shown in Fig. 11 as functions of the neutron number. There is a sharp increase in the $B\left(E 2 ; 9 / 2_{1}^{+} \rightarrow 5 / 2_{1}^{+}\right)$and $B\left(E 2 ; 7 / 2_{1}^{+} \rightarrow\right.$ $5 / 2_{1}^{+}$) strengths in going from $N=88$ to 90 , suggesting a sudden structural change from ${ }^{151} \mathrm{Eu}$ to ${ }^{153} \mathrm{Eu}$, especially in the case of the $7 / 2_{1}^{+} \rightarrow 5 / 2_{1}^{+}$transition. However, our calculations underestimate the experimental $B\left(E 2 ; 5 / 2_{2}^{+} \rightarrow 7 / 2_{1}^{+}\right)$value although they still exhibit a small peak at $N=90$, where the shape transition occurs.

The $B\left(E 2 ; 5 / 2_{1}^{-} \rightarrow 3 / 2_{1}^{-}\right), \quad B\left(E 2 ; 7 / 2_{1}^{-} \rightarrow 3 / 2_{1}^{-}\right)$, and $B\left(E 2 ; 7 / 2_{1}^{-} \rightarrow 5 / 2_{1}^{-}\right)$transition strengths obtained for the odd-mass isotopes ${ }^{149-155} \mathrm{Sm}$ are plotted in Fig. 12 as functions of $N$. Our Gogny-D1M calculations reproduce reasonably well the available data for both the $B\left(E 2 ; 5 / 2_{1}^{-} \rightarrow 3 / 2_{1}^{-}\right)$[panel (a)] and $B\left(E 2 ; 7 / 2_{1}^{-} \rightarrow 3 / 2_{1}^{-}\right)$[panel (b)] strengths in ${ }^{149} \mathrm{Sm}$ and ${ }^{151} \mathrm{Sm}$. Experimental data are not available for the heavier $N=91$ and $N=93$ isotopes.

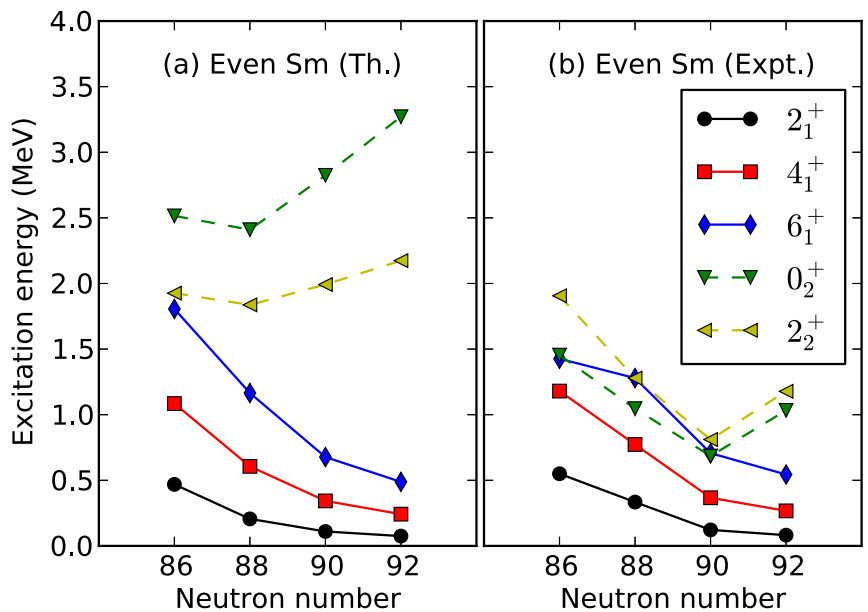

FIG. 7. The low-lying states in ${ }^{148-154} \mathrm{Sm}$ are plotted as functions of the neutron number $N$. Experimental data were taken from Ref. [37]. 


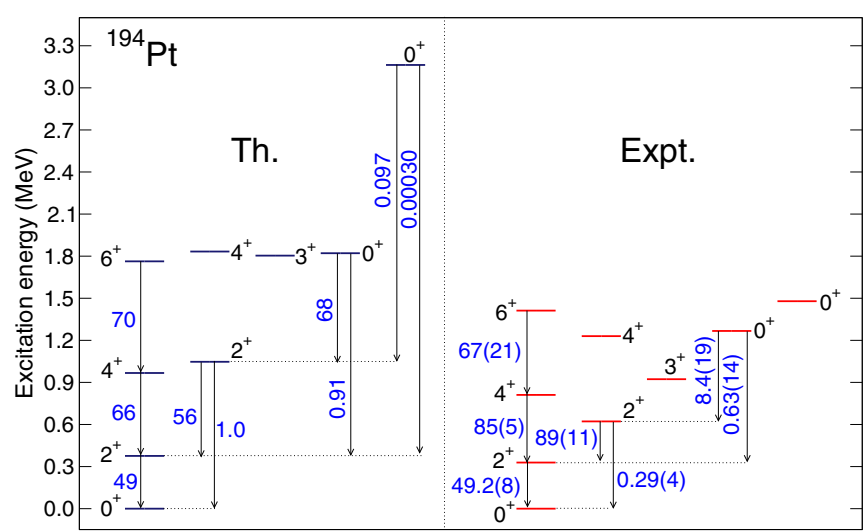

FIG. 8. Energy spectra of ${ }^{194} \mathrm{Pt}$. The numbers along arrows are $B(E 2)$ transition strengths in Weisskopf units. Experimental data have were from Ref. [37].

A sharp rise of the $B\left(E 2 ; 5 / 2_{1}^{-} \rightarrow 3 / 2_{1}^{-}\right)$and $B\left(E 2 ; 7 / 2_{1}^{-} \rightarrow 3 / 2_{1}^{-}\right)$values is predicted from $N=89$ to 91. Such a behavior, as in the case of the odd-mass Eu isotopes (see, Fig. 11), points to a spherical-to-deformed shape transition in the corresponding even-even $\mathrm{Sm}$ nuclei. However, the predicted $B\left(E 2 ; 7 / 2_{1}^{-} \rightarrow 5 / 2_{1}^{-}\right)$transition rate [panel (c)] does not exhibit a clear signature of the rapid structural change as in the other two cases [panels (a) and (b)]. In particular, our calculations underestimate the experimental $B\left(E 2 ; 7 / 2_{1}^{-} \rightarrow 5 / 2_{1}^{-}\right)$value for ${ }^{151} \mathrm{Sm}$. The reason for the disagreement is that the computed wave functions for the $5 / 2_{1}^{-}$and $7 / 2_{1}^{-}$states are very different in nature, i.e., the former is mainly composed of $f_{5 / 2}(29 \%)$ and $h_{9 / 2}(45 \%)$ configurations, whereas the latter is mostly made of the $f_{7 / 2}$ configuration $(79 \%)$.

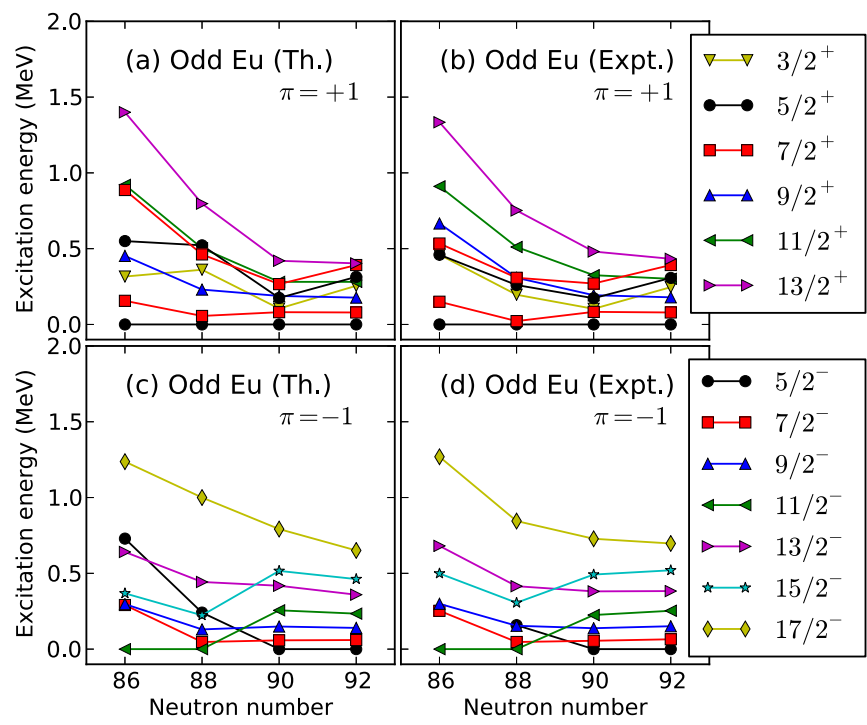

FIG. 9. The low-lying positive- $(\pi=+1)$ and negative-parity $(\pi=-1)$ states in the odd-mass isotopes ${ }^{149-155} \mathrm{Eu}$ are plotted as functions of the neutron number $N$. Experimental data were taken from Ref. [37].

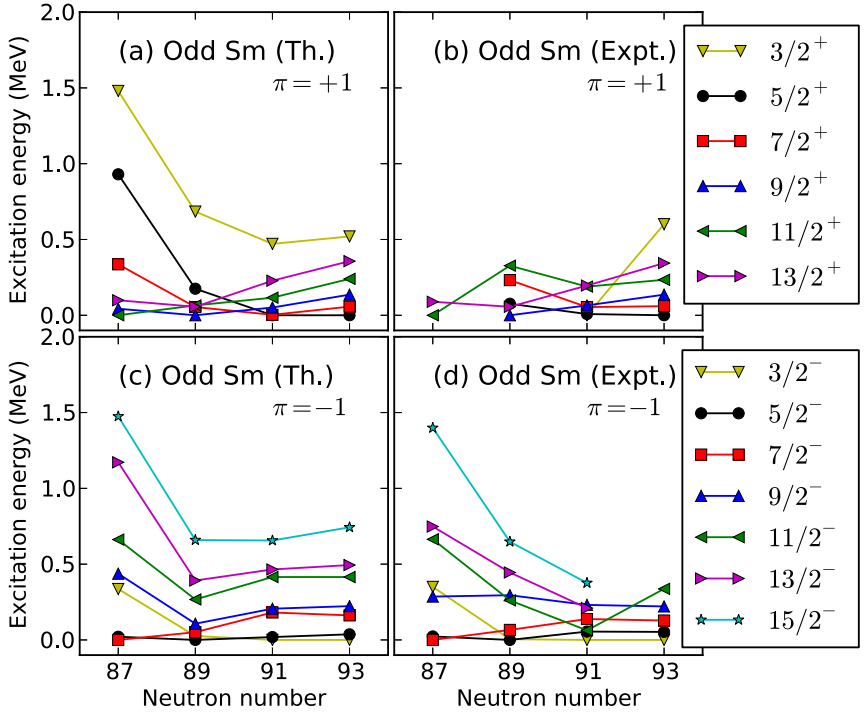

FIG. 10. Same as in Fig. 9, but for the odd-mass Sm isotopes.

Another signature of the shape transition already mentioned can be found in Fig. 13, where the spectroscopic quadrupole $Q_{J}$ and magnetic $\mu_{J}$ moments of the $3 / 2_{1}^{+}, 5 / 2_{1}^{+}, 7 / 2_{1}^{+}, 5 / 2_{1}^{-}$, and $11 / 2_{1}^{-}$states are shown for ${ }^{149-155} \mathrm{Eu}$. Similar to the $B(E 2)$ transition rates, the $Q_{J}$ values in panels (a) to (e) of the figure exhibit a dramatic change around $N=88$ and $N=90$. The agreement between the theoretical and experimental [37,41] $Q_{J}$ values is also very reasonable. On the other hand, the $\mu_{J}$ values, plotted in panels (f) to (j), seem to be less sensitive to $N$ than the $Q_{J}$ ones. One observes a fair agreement between the calculated and experimental $\mu_{J}$ values, except for the

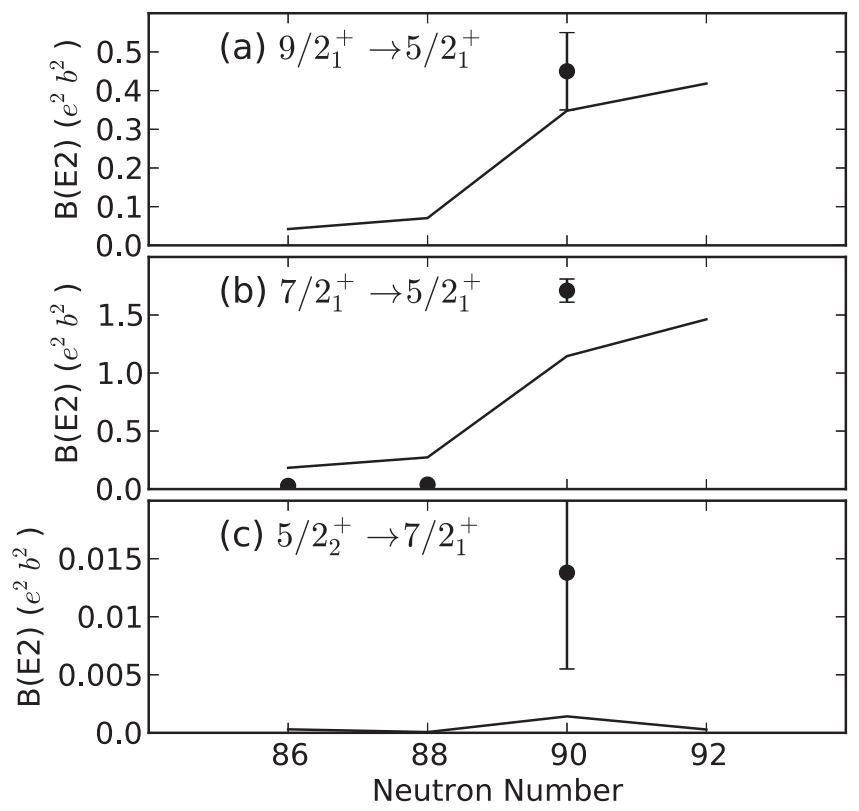

FIG. 11. The $B\left(E 2 ; 9 / 2_{1}^{+} \rightarrow 5 / 2_{1}^{+}\right), B\left(E 2 ; 7 / 2_{1}^{+} \rightarrow 5 / 2_{1}^{+}\right)$, and $B\left(E 2 ; 5 / 2_{2}^{+} \rightarrow 7 / 2_{1}^{+}\right)$transition strengths obtained for the odd-mass isotopes ${ }^{149-155} \mathrm{Eu}$ are depicted as functions of the neutron number $N$. Experimental data were taken from Ref. [37]. 


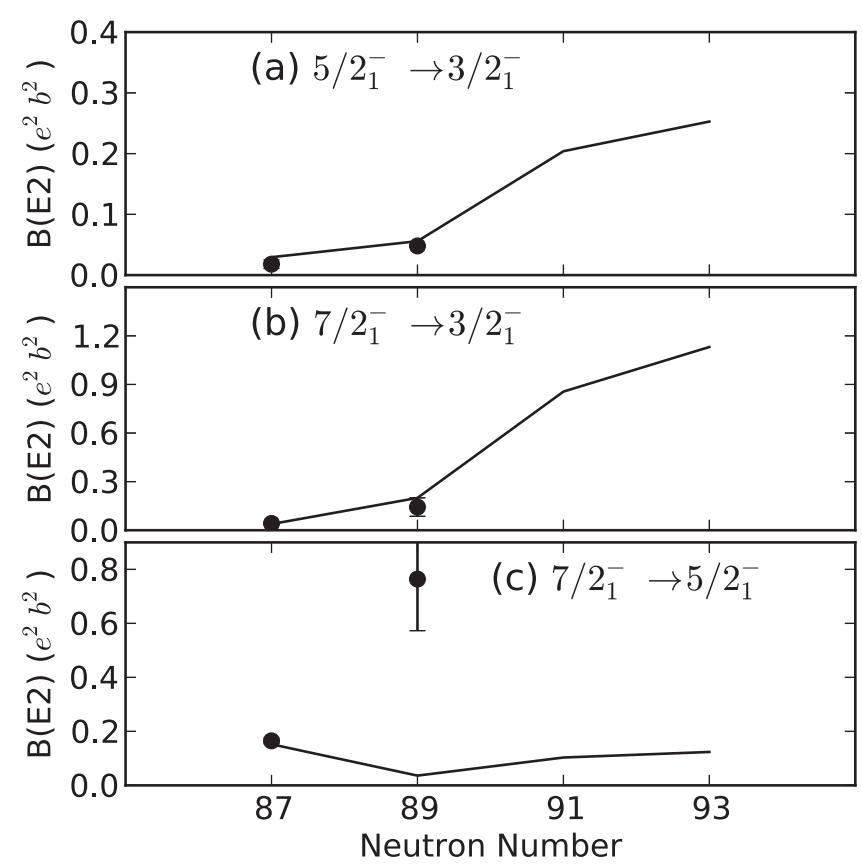

FIG. 12. The $B\left(E 2 ; 5 / 2_{1}^{-} \rightarrow 3 / 2_{1}^{-}\right), B\left(E 2 ; 7 / 2_{1}^{-} \rightarrow 3 / 2_{1}^{-}\right)$, and $B\left(E 2 ; 7 / 2_{1}^{-} \rightarrow 5 / 2_{1}^{-}\right)$transition strengths obtained for the odd-mass isotopes ${ }^{149-155} \mathrm{Sm}$ are depicted as functions of the neutron number $N$. Experimental data were taken from Ref. [37].
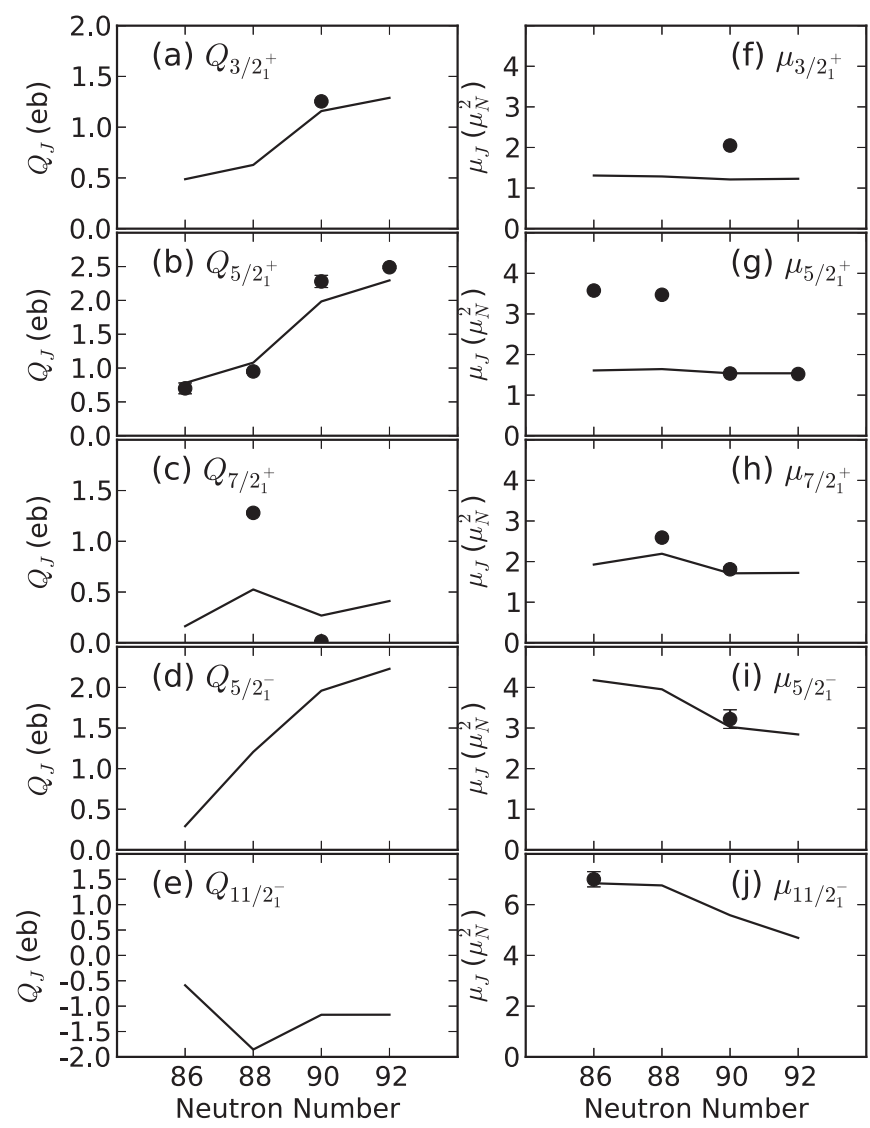

FIG. 13. The spectroscopic quadrupole $Q_{J}$ (in $e$ b units) and magnetic $\mu_{J}$ (in $\mu_{N}^{2}$ units) moments obtained for the odd-mass isotopes ${ }^{149-155} \mathrm{Eu}$ are plotted as functions of the neutron number $N$. Experimental data, represented by dots, were taken from Ref. [41].
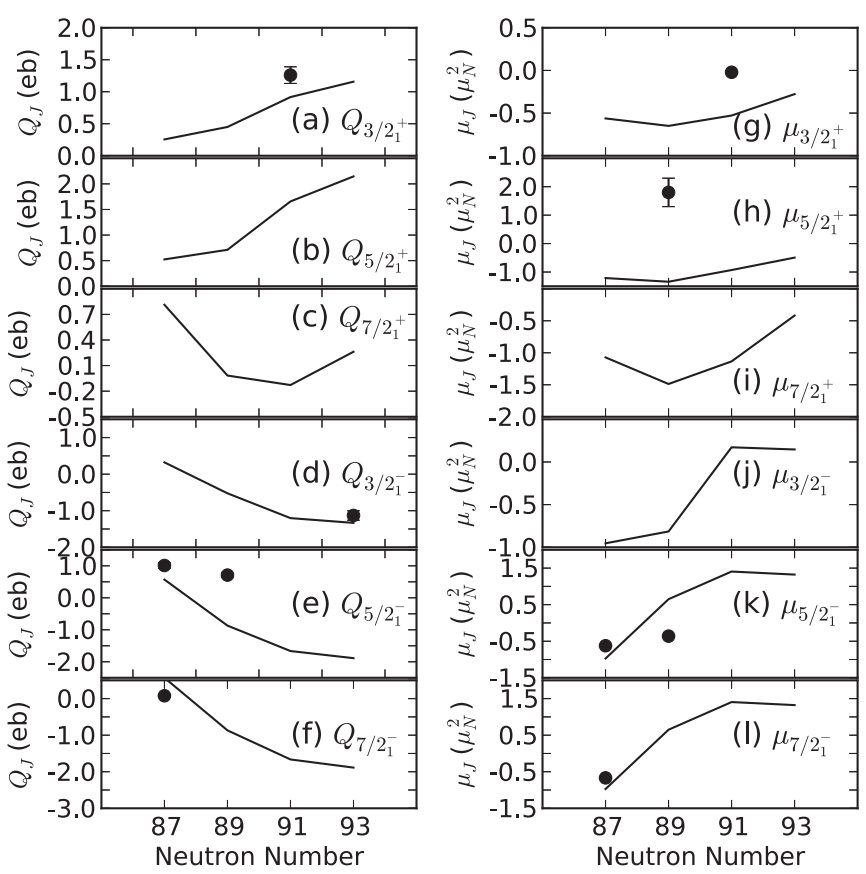

FIG. 14. Same as in Fig. 13, but for the odd-mass isotopes ${ }^{149-155} \mathrm{Sm}$.

substantial disagreement of the $\mu_{5 / 2_{1}^{+}}$values at $N=86$ and $N=88$.

In Fig. 14, we have depicted the $Q_{J}$ and $\mu_{J}$ moments for the $3 / 2_{1}^{ \pm}, 5 / 2_{1}^{ \pm}$, and $7 / 2_{1}^{ \pm}$states in the case of the odd-mass isotopes ${ }^{149-155} \mathrm{Sm}$. As in Fig. 13, the predicted $Q_{J}$ values [panels (a) to (f)] exhibit a rapid change around $N=89$ or $N=$ 91, where the shape transition occurs. Notice, that the sign of $Q_{3 / 2_{1}^{-}}$for ${ }^{155} \mathrm{Sm}$ is not known experimentally [37,41], though it is assumed to be negative for consistency with the calculated one. On the other hand, many of the predicted $\mu_{J}$ values [panels (g) to (1)] also exhibit a significant change around the transitional system with $N=89$ or $N=91$. In many cases, however, the sign of the corresponding $\mu_{J}$ moments is opposite to the experimental one.

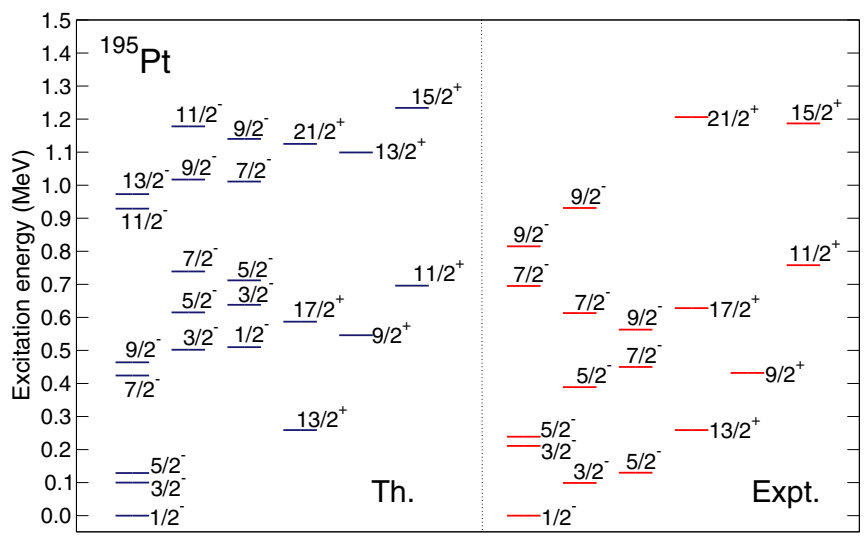

FIG. 15. The three lowest positive- and negative-parity bands in ${ }^{195} \mathrm{Pt}$. Experimental data were taken from Ref. [37]. 


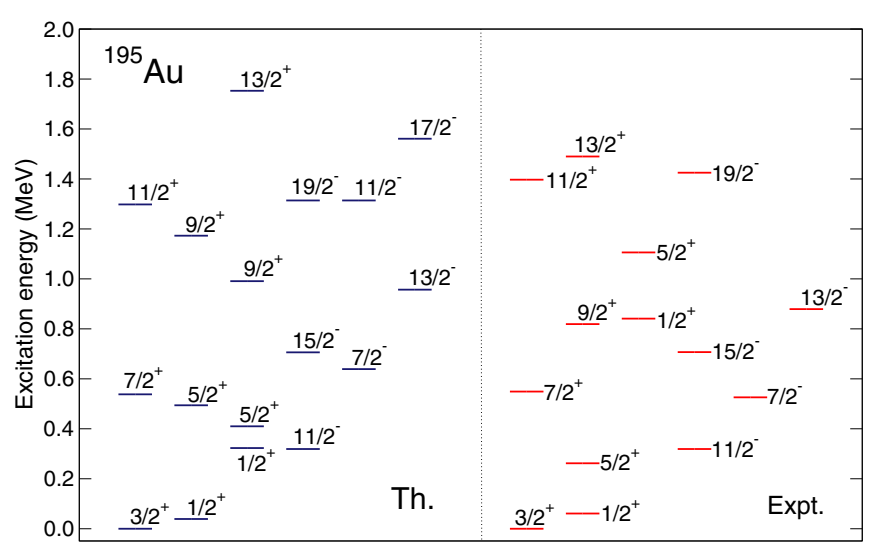

FIG. 16. Same as in Fig. 15, but for ${ }^{195} \mathrm{Au}$.

\section{SPECTROSCOPIC PROPERTIES OF ${ }^{195}$ Pt AND ${ }^{195} \mathrm{Au}$}

In this section we turn our attention to the $\gamma$-soft cases. In Figs. 15 and 16 we have plotted the three lowest positiveand negative-parity bands obtained for both ${ }^{195} \mathrm{Pt}$ and ${ }^{195} \mathrm{Au}$. They are compared with the experimental ones taken from Ref. [37]. To understand the structure of those states, we show in Tables V and VI the amplitudes of their decomposition in the spherical single particle basis. The two tables correspond to the results for ${ }^{195} \mathrm{Pt}$ and ${ }^{195} \mathrm{Au}$, respectively. It should be noted that, in Figs. 15 and 16, the theoretical levels have been classified into bands according to the dominant $E 2$ decays and also that the relative location of the ground states for positive and negative parity in the theoretical energy spectrum has been adjusted to that of the experimental data.

As can be seen from Fig. 15, our calculations reproduce fairly well the experimental energies of the negative-parity states in ${ }^{195} \mathrm{Pt}$, whereas many of the non-yrast states are

TABLE V. Amplitudes (in percent) of the negative-parity states of ${ }^{195} \mathrm{Pt}$ shown in Fig. 15 when expressed in the single-particle basis of the $3 p_{1 / 2}, 3 p_{3 / 2}, 2 f_{5 / 2}, 2 f_{7 / 2}$, and $1 h_{9 / 2}$ orbitals.

\begin{tabular}{lrrrrr}
\hline \hline$J^{\pi}$ & $3 p_{1 / 2}$ & $3 p_{3 / 2}$ & $2 f_{5 / 2}$ & $2 f_{7 / 2}$ & $1 h_{9 / 2}$ \\
\hline $1 / 2_{1}^{-}$ & 67 & 12 & 12 & 5 & 4 \\
$1 / 2_{2}^{-}$ & 0 & 46 & 39 & 8 & 7 \\
$3 / 2_{1}^{-}$ & 51 & 31 & 5 & 11 & 2 \\
$3 / 2_{2}^{-}$ & 0 & 28 & 57 & 7 & 8 \\
$3 / 2_{3}^{-}$ & 16 & 40 & 31 & 7 & 6 \\
$5 / 2_{1}^{-}$ & 53 & 6 & 30 & 3 & 8 \\
$5 / 2_{2}^{-}$ & 15 & 22 & 50 & 6 & 7 \\
$5 / 2_{3}^{-}$ & 3 & 51 & 30 & 11 & 5 \\
$7 / 2_{1}^{-}$ & 44 & 34 & 7 & 13 & 2 \\
$7 / 2_{2}^{-}$ & 4 & 16 & 66 & 4 & 10 \\
$7 / 2_{3}^{-}$ & 19 & 44 & 23 & 10 & 4 \\
$9 / 2_{1}^{-}$ & 47 & 5 & 35 & 2 & 11 \\
$9 / 2_{2}^{-}$ & 19 & 19 & 48 & 4 & 9 \\
$9 / 2_{3}^{-}$ & 2 & 49 & 32 & 12 & 4 \\
$11 / 2_{1}^{-}$ & 42 & 34 & 8 & 14 & 2 \\
$11 / 2_{2}^{-}$ & 3 & 14 & 68 & 3 & 12 \\
$13 / 2_{1}^{-}$ & 45 & 5 & 37 & 2 & 11 \\
\hline \hline
\end{tabular}

TABLE VI. Amplitudes (in percent) of the positive-parity states of ${ }^{195} \mathrm{Au}$ shown in Fig. 16 when expressed in the single-particle basis of the $3 s_{1 / 2}, 2 d_{3 / 2}, 2 d_{5 / 2}$, and $1 g_{7 / 2}$ orbitals.

\begin{tabular}{lcccc}
\hline \hline$J^{\pi}$ & $3 s_{1 / 2}$ & $2 d_{3 / 2}$ & $2 d_{5 / 2}$ & $1 g_{7 / 2}$ \\
\hline $1 / 2_{1}^{+}$ & 59 & 27 & 12 & 2 \\
$1 / 2_{2}^{+}$ & 14 & 61 & 15 & 10 \\
$3 / 2_{1}^{+}$ & 3 & 84 & 2 & 11 \\
$5 / 2_{1}^{+}$ & 1 & 83 & 6 & 10 \\
$5 / 2_{2}^{+}$ & 67 & 11 & 21 & 1 \\
$7 / 2_{1}^{+}$ & 2 & 83 & 1 & 14 \\
$9 / 2_{1}^{+}$ & 8 & 73 & 9 & 10 \\
$9 / 2_{2}^{+}$ & 60 & 19 & 20 & 1 \\
$11 / 2_{1}^{+}$ & 1 & 83 & 2 & 14 \\
$13 / 2_{1}^{+}$ & 14 & 68 & 9 & 9 \\
\hline \hline
\end{tabular}

overestimated. Both theoretically and experimentally, the lowest negative-parity band appears to show the $\Delta J=1$ systematics of the strong coupling limit, though there is a staggering pattern $\left(3 / 2^{-}, 5 / 2^{-}\right),\left(7 / 2^{-}, 9 / 2^{-}\right)$, etc. Consistent with the experiment, the first and second excited negativeparity bands also display a $\Delta J=1$ feature. Nevertheless, their bandhead energies are rather high in comparison with the experimental ones. Furthermore, we have classified the calculated $3 / 2_{1}^{-}$and $5 / 2_{1}^{-}$levels, which are nearly degenerate, into the lowest band, whereas the experimental $3 / 2_{1}^{-}$and $5 / 2_{1}^{-}$ levels are suggested to be the bandheads of the first and second excited bands, respectively. As seen from Table V. the features already mentioned can be understood from the fact that, in our calculations, the states in the lowest negative-parity band are predominantly composed of the $p_{1 / 2}$ configuration while those in the first and second excited negative-parity bands are mainly composed of the $f_{5 / 2}$ and $p_{3 / 2}$ configurations, respectively. From Table II we note that the $3 p_{3 / 2}$ and $2 f_{5 / 2}$ single-particle levels lie much higher than the $3 p_{1 / 2}$ orbital. This could partly account for the discrepancy observed in ${ }^{195} \mathrm{Pt}$ where the first and second excited negative-parity bands are predicted to lie too high in excitation energy as compared to the experiment. On the other hand, the pattern of the positive-parity levels in ${ }^{195} \mathrm{Pt}$, also shown in Fig. 15, is much simpler than for the negative-parity ones. The ground state for positive parity, the $13 / 2_{1}^{+}$state, is weakly coupled to the boson core nucleus ${ }^{194} \mathrm{Pt}$. Consistent with the experiment, the three theoretical positive-parity bands, shown in the left-hand side of Fig. 15, look rather harmonic. They exhibit the weak coupling $\Delta J=2$ systematics.

The results obtained for ${ }^{195} \mathrm{Au}$ are shown in Fig. 16. In this case, our calculations provide a slightly better agreement with the experimental data than for ${ }^{195} \mathrm{Pt}$. The three lowest positive-parity bands display the $\Delta J=2$ systematics of the weak coupling limit. This is consistent with the experimental data. Nevertheless, the calculated first excited positive-parity band looks more stretched than the experimental one. Also, the second excited positive-parity band, built on the $1 / 2_{2}^{+}$state, is much lower in energy in the present calculation than in experiment. In our calculation, the low-lying positive-parity states of the ${ }^{195} \mathrm{Au}$ nucleus are mainly composed of the $s_{1 / 2}$ and 
TABLE VII. $B(E 2)$ and $B(M 1)$ transition probabilities (in Weisskopf units) for ${ }^{195} \mathrm{Pt}$.

\begin{tabular}{|c|c|c|c|c|}
\hline & \multicolumn{2}{|c|}{$B(E 2)$ (W.u.) } & \multicolumn{2}{|c|}{$B(M 1)$ (W.u.) } \\
\hline & Th. & Expt. & Th. & Expt. \\
\hline $3 / 2_{1}^{-} \rightarrow 1 / 2_{1}^{-}$ & 36 & $11.5(15)$ & $3.9 \times 10^{-5}$ & $0.0168(19)$ \\
\hline $3 / 2_{2}^{-} \rightarrow 1 / 2_{1}^{-}$ & 0.086 & $4.5(13)$ & 0.00032 & $0.00033(11)$ \\
\hline $3 / 2_{3}^{-} \rightarrow 1 / 2_{1}^{-}$ & 6.1 & $30(7)$ & 0.023 & $0.024(4)$ \\
\hline $3 / 2_{4}^{-} \rightarrow 1 / 2_{1}^{-}$ & 2.9 & $0.22(7)$ & 0.011 & $0.0036(7)$ \\
\hline $3 / 2_{4}^{-} \rightarrow 1 / 2_{2}^{-}$ & 4.2 & $<37$ & 0.016 & $>0.00054$ \\
\hline $5 / 2_{1}^{-} \rightarrow 1 / 2_{1}^{-}$ & 35 & $8.9(7)$ & & \\
\hline $5 / 2_{2}^{-} \rightarrow 1 / 2_{1}^{-}$ & 7.5 & $49(7)$ & & \\
\hline $5 / 2_{3}^{-} \rightarrow 1 / 2_{1}^{-}$ & 0.0093 & $1.3(9)$ & & \\
\hline $3 / 2_{2}^{-} \rightarrow 3 / 2_{1}^{-}$ & 4.0 & $0.05_{-5}^{+106}$ & 0.0043 & $0.0030(8)$ \\
\hline $3 / 2_{4}^{-} \rightarrow 3 / 2_{1}^{-}$ & 6.8 & $0.07(6)$ & $4.3 \times 10^{-5}$ & $0.0013(3)$ \\
\hline $5 / 2_{1}^{-} \rightarrow 3 / 2_{1}^{-}$ & 9.9 & $4.8(19)$ & 0.017 & $0.0269(21)$ \\
\hline $5 / 2_{2}^{-} \rightarrow 3 / 2_{1}^{-}$ & 0.076 & $11(6)$ & $9.9 \times 10^{-5}$ & $0.019(3)$ \\
\hline $5 / 2_{3}^{-} \rightarrow 3 / 2_{1}^{-}$ & 7.2 & $38(20)$ & 0.027 & $0.038(17)$ \\
\hline $5 / 2_{4}^{-} \rightarrow 3 / 2_{1}^{-}$ & & & 0.0097 & $<0.013$ \\
\hline $5 / 2_{4}^{-} \rightarrow 3 / 2_{3}^{-}$ & & & 0.0030 & $<0.017$ \\
\hline $7 / 2_{2}^{-} \rightarrow 3 / 2_{1}^{-}$ & 0.84 & $29(10)$ & & \\
\hline $7 / 2_{2}^{-} \rightarrow 3 / 2_{3}^{-}$ & 4.0 & $7(3)$ & & \\
\hline $7 / 2_{3}^{-} \rightarrow 3 / 2_{3}^{-}$ & 34 & $26(17)$ & & \\
\hline $5 / 2_{3}^{-} \rightarrow 5 / 2_{1}^{-}$ & 1.9 & $0.015_{-15}^{+88}$ & 0.0044 & $0.026(12)$ \\
\hline $7 / 2_{2}^{-} \rightarrow 5 / 2_{1}^{-}$ & & & 0.033 & $0.014(5)$ \\
\hline $9 / 2_{1}^{-} \rightarrow 5 / 2_{1}^{-}$ & 57 & $35(8)$ & & \\
\hline $5 / 2_{3}^{-} \rightarrow 5 / 2_{2}^{-}$ & & & 0.027 & $0.030(15)$ \\
\hline $5 / 2_{4}^{-} \rightarrow 5 / 2_{2}^{-}$ & 6.3 & $<60$ & & \\
\hline $7 / 2_{3}^{-} \rightarrow 5 / 2_{2}^{-}$ & 1.7 & $<210$ & 0.0064 & $<0.077$ \\
\hline $9 / 2_{2}^{-} \rightarrow 5 / 2_{2}^{-}$ & 47 & $30(8)$ & & \\
\hline $7 / 2_{2}^{-} \rightarrow 5 / 2_{3}^{-}$ & 2.1 & $<3.9 \times 10^{3}$ & 0.0080 & $<0.14$ \\
\hline
\end{tabular}

$d_{3 / 2}$ configurations. For example, $84 \%$ of the ground state $3 / 2_{1}^{+}$ is made of the $d_{3 / 2}$ configuration, while $59 \%$ of the first excited state $1 / 2_{1}^{+}$is comprised of the $s_{1 / 2}$ configuration (see Table VI). Similarly to the positive-parity bands in ${ }^{195} \mathrm{Pt}$ nucleus, the theoretical negative-parity bands in ${ }^{195} \mathrm{Au}$ all exhibit the weakcoupling $\Delta J=2$ systematics and look harmonic.

The calculated $B(E 2)$ and $B(M 1)$ transition rates of the ${ }^{195} \mathrm{Pt}$ nucleus are shown in Table VII and compared with the experimental data of Ref. [37,41]. As is apparent from the table, the agreement between our calculation and experiment is not necessarily satisfactory in some of the $B(E 2)$ transition rates. For instance, the theoretical $B\left(E 2 ; 5 / 2_{2}^{-} \rightarrow 3 / 2_{1}^{-}\right)$value of 0.076 W.u. is roughly a factor $10^{2}$ smaller than the experimental value of $11 \pm 6$ W.u. A possible reason for the disagreement could be attributed to the choice of both the boson and fermion effective charges. A more likely reason would be the fact that the structures of the $5 / 2_{2}^{-}$and $3 / 2_{1}^{-}$wave functions are somewhat different in the present calculation (see Table V). The $3 / 2_{1}^{-}$state is mostly composed of the $p_{1 / 2}(51 \%)$ and $p_{3 / 2}(31 \%)$ configurations. On the other hand, the main component of the $5 / 2_{2}^{-}$wave function is the $f_{5 / 2}$ configuration $(50 \%)$, while the $p_{1 / 2}$ and $p_{3 / 2}$ configurations account for $15 \%$ and $22 \%$ of the wave function, respectively. All in all, the predicted $B(M 1)$ values compare well with the experimental data. We also compare in Table VIII the
TABLE VIII. Spectroscopic quadrupole (in $e b$ ) and magnetic moments $\left(\right.$ in $\left.\mu_{N}^{2}\right)$ in ${ }^{195} \mathrm{Pt}$.

\begin{tabular}{lccccc}
\hline \hline & \multicolumn{2}{c}{$Q_{J}(e \mathrm{~b})$} & & \multicolumn{2}{c}{$\mu_{J}\left(\mu_{N}^{2}\right)$} \\
\cline { 2 - 3 } \cline { 5 - 6 } & Th. & Expt. & & Th. & Expt. \\
\hline $13 / 2_{1}^{+}$ & +1.619 & $+1.4(6)$ & & -1.246 & $-0.606(105)$ \\
$1 / 2_{1}^{-}$ & & & +0.467 & $+0.60952(6)$ \\
$3 / 2_{1}^{-}$ & +0.683 & & & -0.206 & $-0.62(6)$ \\
$3 / 2_{3}^{-}$ & +0.288 & & & -0.411 & $+0.16(3)$ \\
$5 / 2_{1}^{-}$ & +0.973 & & +0.924 & $+0.90(6)$ \\
$5 / 2_{2}^{-}$ & +0.306 & & +1.219 & $+0.52(5)$ \\
$5 / 2_{3}^{-}$ & +0.536 & & +0.201 & $+0.39(10)$ \\
$5 / 2_{4}^{-}$ & -0.729 & & +0.925 & $+1.6(6)$ \\
$7 / 2_{2}^{-}$ & +0.637 & & +1.079 & $+0.55(8)$ \\
$7 / 2_{3}^{-}$ & +0.566 & & +0.270 & $+1.4(4)$ \\
$7 / 2_{5}^{-}$ & +0.179 & & +0.212 & $+1.2(3)$ \\
$9 / 2_{2}^{-}$ & +0.731 & & +1.735 & $+1.55(12)$ \\
$9 / 2_{3}^{-}$ & +0.744 & +0.785 & $+1.52(16)$ \\
\hline \hline
\end{tabular}

predicted and experimental [37,41] spectroscopic quadrupole $Q_{J}$ and magnetic $\mu_{J}$ moments of the ${ }^{195} \mathrm{Pt}$ nucleus. In this table, the calculated $Q_{13 / 2^{+}}$value is in good agreement with the experimental one. We also show in Table VIII the predicted $Q_{J}$ values for other states, where data are not available. The sign of the predicted $\mu_{J}$ values is, in most cases, consistent with the data, apart from $\mu_{3 / 2_{3}^{-}}$.

In Table IX we compare the calculated $B(E 2), B(M 1), Q_{J}$ and $\mu_{J}$ values of the ${ }^{195} \mathrm{Au}$ nucleus with the experimental data. Overall, the calculation reproduces experimental data rather well. As mentioned above, some disagreement between the calculated and experimental $B(E 2)$ values could partly arise from the chosen effective charges for the fermion and boson quadrupole operators, although it is more likely to be due to the differences in the nature of the wave functions of the initial and final states. In the case of the $B\left(E 2 ; 5 / 2_{1}^{+} \rightarrow 3 / 2_{2}^{+}\right)$transition rate, for instance, the wave function of the $5 / 2_{1}^{+}$state in ${ }^{195} \mathrm{Au}$ is made predominantly of the $d_{3 / 2}$ single-particle configuration ( $83 \%$ ), while the $3 / 2_{2}^{+}$wave function is mainly composed of the $s_{1 / 2}$ configuration (66\%). For more details, see Table VI.

TABLE IX. $B(E 2)$ and $B(M 1)$ transition strengths, and spectroscopic quadrupole and magnetic moments in ${ }^{195} \mathrm{Au}$.

\begin{tabular}{|c|c|c|c|c|}
\hline & \multicolumn{2}{|c|}{$B(E 2)$ (W.u.) } & \multicolumn{2}{|c|}{$B(M 1)$ (W.u.) } \\
\hline & Th. & Expt. & Th. & Expt. \\
\hline \multirow{8}{*}{$\begin{array}{l}1 / 2_{1}^{+} \rightarrow 3 / 2_{1}^{+} \\
3 / 2_{2}^{+} \rightarrow 1 / 2_{1}^{+} \\
3 / 2_{2}^{+} \rightarrow 3 / 2_{1}^{+} \\
5 / 2_{1}^{+} \rightarrow 3 / 2_{2}^{+} \\
5 / 2_{1}^{+} \rightarrow 3 / 2_{1}^{+} \\
25 / 2_{1}^{+} \rightarrow 21 / 2_{1}^{+}\end{array}$} & 31 & $41(4)$ & 0.102 & $0.00199(15)$ \\
\hline & 31 & $>15$ & 0.114 & $>0.051$ \\
\hline & 1.4 & $>4.3$ & 0.0062 & $>0.00013$ \\
\hline & 0.62 & $8.7(20)$ & & \\
\hline & 39 & $18(4)$ & $9.3 \times 10^{-5}$ & $0.0124(25)$ \\
\hline & 41 & $10.9(25)$ & & \\
\hline & & $Q_{J}(e \mathrm{~b})$ & $\mu_{J}$ & $\left(\mu_{N}^{2}\right)$ \\
\hline & Th. & Expt. & Th. & Expt. \\
\hline$\overline{3 / 2_{1}^{+}}$ & +0.781 & $+0.607(18)$ & +0.633 & $+0.145(5)$ \\
\hline $11 / 2_{1}^{-}$ & +1.708 & $+1.87(6)$ & +6.70 & $+6.17(9)$ \\
\hline
\end{tabular}


One realizes in Table IX that the calculated $Q_{J}$ and $\mu_{J}$ values for the lowest positive- $\left(3 / 2_{1}^{+}\right)$and negative-parity $\left(11 / 2_{1}^{-}\right)$ states of the ${ }^{195} \mathrm{Au}$ nucleus are in an excellent agreement with the available experimental data $[37,41]$.

\section{SUMMARY AND CONCLUDING REMARKS}

In this work we studied the spectroscopic properties of several odd-mass nuclei within the IBFM framework based on the Gogny-D1M EDF. Following the procedure developed in Ref. [21], the $(\beta, \gamma)$-deformation energy surface for the even-even core nuclei, as well as the single-particle energies and occupation probabilities of the odd nucleon, were provided by the self-consistent HFB method based on the Gogny-D1M $\mathrm{EDF}$, and were used as a microscopic input for the construction of the Hamiltonian of the IBFM. As was done in the original work of Ref. [21], the three strength parameters of the particlecore coupling Hamiltonian were fitted in each of the odd-mass nucleus considered, to reproduce selected experimental data for the low-energy excitation spectra. The method was applied to the axially deformed odd-mass ${ }^{149-155} \mathrm{Eu}$ and ${ }^{149-155} \mathrm{Sm}$ nuclei, and to the $\gamma$-soft odd-mass ${ }^{195} \mathrm{Pt}$ and ${ }^{195} \mathrm{Au}$ nuclei.

The present calculation describes fairly well the experimental systematics of excitation spectra and electromagnetic properties of the odd-mass $\mathrm{Eu}$ and $\mathrm{Sm}$ nuclei as signatures of structural evolution from the nearly spherical to axially deformed shapes. Our calculation on the odd-mass Eu and Sm nuclei reveals the same level of accuracy in describing the odd- mass isotopes as the previous calculations on the same nuclei $[21,23]$ based on a relativistic EDF. The method also provides a reasonable description of the low-energy excitation spectra in the $\gamma$-soft ${ }^{195} \mathrm{Pt}$ and ${ }^{195} \mathrm{Au}$ nuclei, whereas the agreement between the calculated and experimental electromagnetic properties is not entirely satisfactory. Nevertheless, considering the fact the model involves only three phenomenological parameters, all these results for the $\gamma$-soft systems seem to be rather promising.

Our next step would be to apply the method to study more systematically the structural evolution in other $\gamma$-soft odd-mass systems, either because they are supposed to have a rich spectrum and/or are of experimental interest. A more ambitious perspective is the determination of the bosonfermion coupling Hamiltonian parameters from quantities obtained solely from the mean field calculations with the Gogny EDF. This possibility will give us the key to make true predictions for odd- $A$ systems where no experimental data exist. Work along these lines is in progress and will be reported elsewhere.

\section{ACKNOWLEDGMENTS}

We would like to thank D. Vretenar and T. Nikšić for useful discussions. K.N. acknowledges support from the Japan Society for the Promotion of Science. This work has been supported in part by the QuantiXLie Centre of Excellence. The work of L.M.R. was supported by Spanish Grants No. FPA2015-65929-P MINECO and No. FIS2015-63770-P MINECO.
[1] A. Bohr and B. M. Mottelsson, Nuclear Structure, Vol. 2 (Benjamin, New York, 1975), p. 45.

[2] A. Bohr, Mat. Fys. Medd. K. Dan. Vidensk. Selsk. 27, 16 (1953).

[3] E. Caurier, G. Martínez-Pinedo, F. Nowacki, A. Poves, and A. P. Zuker, Rev. Mod. Phys. 77, 427 (2005).

[4] M. Bender, P.-H. Heenen, and P.-G. Reinhard, Rev. Mod. Phys. 75, 121 (2003).

[5] L. M. Robledo, R. Bernard, and G. F. Bertsch, Phys. Rev. C 86, 064313 (2012).

[6] R. Rodríguez-Guzmán, P. Sarriguren, L. M. Robledo, and S. Perez-Martin, Phys. Lett. B 691, 202 (2010).

[7] R. Rodríguez-Guzmán, P. Sarriguren, and L. M. Robledo, Phys. Rev. C 82, 044318 (2010).

[8] R. Rodríguez-Guzmán, P. Sarriguren, and L. M. Robledo, Phys. Rev. C 82, 061302 (2010).

[9] B. Bally, B. Avez, M. Bender, and P.-H. Heenen, Phys. Rev. Lett. 113, 162501 (2014).

[10] M. Borrajo and J. L. Egido, Eur. Phys. J. A 52, 277 (2016).

[11] E. V. Litvinova and A. V. Afanasjev, Phys. Rev. C 84, 014305 (2011).

[12] K. Mizuyama, G. Colò, and E. Vigezzi, Phys. Rev. C 86, 034318 (2012).

[13] Y. F. Niu, Z. M. Niu, G. Colò, and E. Vigezzi, Phys. Rev. Lett. 114, 142501 (2015).
[14] G. De Gregorio, F. Knapp, N. Lo Iudice, and P. Vesely, Phys. Rev. C 94, 061301 (2016).

[15] G. De Gregorio, F. Knapp, N. Lo Iudice, and P. Veselý, Phys. Rev. C 95, 034327 (2017).

[16] O. Scholten, Prog. Part. Nucl. Phys. 14, 189 (1985).

[17] F. Iachello and P. Van Isacker, The Interacting Boson-Fermion Model (Cambridge University Press, Cambridge, 1991).

[18] Interacting Bose-Fermi Systems in Nuclei, edited by F. Iachello (Springer, New York, 1981).

[19] F. Iachello, A. Leviatan, and D. Petrellis, Phys. Lett. B 705, 379 (2011).

[20] D. Petrellis, A. Leviatan, and F. Iachello, Ann. Phys. (N.Y.) 326, 926 (2011).

[21] K. Nomura, T. Nikšić, and D. Vretenar, Phys. Rev. C 93, 054305 (2016)

[22] T. Nikšić, D. Vretenar, and P. Ring, Phys. Rev. C 78, 034318 (2008).

[23] K. Nomura, T. Nikšić, and D. Vretenar, Phys. Rev. C 94, 064310 (2016).

[24] K. Nomura, T. Nikšić, and D. Vretenar, Phys. Rev. C 96, 014304 (2017).

[25] S. Goriely, S. Hilaire, M. Girod, and S. Péru, Phys. Rev. Lett. 102, 242501 (2009).

[26] J. F. Berger, M. Girod, and D. Gogny, Nucl. Phys. A 428, 23 (1984). 
[27] T. Otsuka, A. Arima, and F. Iachello, Nucl. Phys. A 309, 1 (1978).

[28] K. Nomura, N. Shimizu, and T. Otsuka, Phys. Rev. Lett. 101, 142501 (2008).

[29] K. Nomura, N. Shimizu, and T. Otsuka, Phys. Rev. C 81, 044307 (2010).

[30] K. Nomura, T. Otsuka, N. Shimizu, and L. Guo, Phys. Rev. C 83, 041302 (2011).

[31] J. N. Ginocchio and M. W. Kirson, Nucl. Phys. A 350, 31 (1980).

[32] L. M. Robledo, R. R. Rodríguez-Guzmán, and P. Sarriguren, Phys. Rev. C 78, 034314 (2008).

[33] R. Rodríguez-Guzmán, P. Sarriguren, L. M. Robledo, and J. E. García-Ramos, Phys. Rev. C 81, 024310 (2010).
[34] T. Otsuka and N. Yoshida, JAERI-M (Japan Atomic Energy Research Institute) Report No. 85 (1985).

[35] F. Iachello and A. Arima, The Interacting Boson Model (Cambridge University Press, Cambridge, 1987).

[36] O. Scholten and N. Blasi, Nucl. Phys. A 380, 509 (1982).

[37] Brookhaven National Laboratory, National Nuclear Data Center, http://www.nndc.bnl.gov.

[38] P. Cejnar, J. Jolie, and R. F. Casten, Rev. Mod. Phys. 82, 2155 (2010).

[39] F. Iachello, Phys. Rev. Lett. 87, 052502 (2001).

[40] R. F. Casten and N. V. Zamfir, Phys. Rev. Lett. 87, 052503 (2001).

[41] N. Stone, At. Data Nucl. Data Tables 90, 75 (2005). 